\title{
LCDs Versus CRTs-Color-Calibration and Gamut Considerations
}

\author{
GAURAV SHARMA, SENIOR MEMBER, IEEE
}

Invited Paper

This paper presents a comparative evaluation of liquid-crystal display (LCDs) and cathode-ray tube (CRT) displays from a color-rendition and color-calibration perspective. Common display calibration models and assumptions are reviewed and their applicability to LCDs and CRTs is evaluated through an experimental study. The displays are compared with respect to the color-calibration accuracy, ease of calibration, and achievable color gamut. The offset, matrix, and tone-response correction model commonly employed for CRT color calibration is also suitable for color calibration of LCDs for most applications, though the calibration error for LCDs is higher. For the prototype LCDs used in the experimental study, large color variations significantly above the calibration accuracy are observed with changes in viewing angle. Under typical viewing conditions, LCDs provide a significantly larger color gamut than CRTs primarily due to their higher luminances.

Keywords-Color calibration, CRT, CRT gamut, display color gamut, LCD, liquid-crystal displays.

\section{INTRODUCTION}

Liquid-crystal display (LCD) flat panels are becoming increasingly common as computer color displays due to their compact size and low power consumption. These displays are now available at increasingly higher spatial resolutions and in larger screen sizes with image quality that meets or exceeds that of typical cathode-ray tube (CRT) displays [1]. While the market for CRTs continues to grow at present, in the long run, flat-panel displays are expected to replace CRTs as the primary computer displays [2], [3].

With the widespread use, there is also an increased need for color management for LCDs, which enables accurate control of color in displayed images. While the color characteristics of CRT displays and methods for their color calibration have been extensively studied and reported [4]-[9], the color characteristics of LCDs and methods for

Manuscript received November 26, 2000; revised August 13, 2001.

The author is with the Digital Imaging Technology Center, Xerox Corporation, Webster, NY 14580 USA (e-mail: g.sharma@ieee.org).

Publisher Item Identifier S 0018-9219(02)03960-9. calibration have only come to the forefront in the last few years and have received only limited attention in published literature [10]-[12].

Active-matrix LCDs (AMLCDs) represent the most commonly employed LCD technology for computer displays. This paper reviews the color characteristics and the color-calibration requirements for AMLCDs and contrasts them with CRT displays which represent the predominant display technology employed today. Common physical assumptions and models for display color calibration are first reviewed in Section II. The specializations of these models to CRT displays are summarized in Section III. The applicability of the models to the AMLCDs in light of their operational physics is considered in Section IV. Sections V-VIII present experimental calibration results for a prototype AMLCD [13] along with corresponding results for a CRT display. Color-calibration models, calibration accuracy, dynamic range, and achievable color gamuts for LCDs and CRTs are compared and contrasted in Section IX. The major conclusions emerging from the comparative study are summarized in Section X.

\section{DisPlay COLOR-CALIBRATION MODELS}

In order to consider the color calibration of displays, it is useful to consider a mathematical model that represents their operation. A general mathematical framework for device calibration has been described in [14]. This section will focus on specifics and details applicable to display calibration.

The display is driven by a set of control signals, typically in the form of an R, G, B triplet for each pixel corresponding, respectively, to the red, green, and blue channels for that pixel. In the most general case, the light emitted by a pixel location could be a function of the present and previous history of driving signals for the entire set of pixels on the display. Clearly, a model of this generality would be too difficult to characterize and use in color calibration and, therefore, simplifying assumptions and corresponding simpler models are 
employed in practice. These assumptions and models are reviewed in this section. Comments on the practical validity of these assumptions for CRTs and LCDs are included in later sections.

Typically, the (small amount of) coupling between neighboring pixel elements is ignored and, in addition, the display is assumed to be spatially homogeneous and temporally stable, i.e., it is assumed that the light emitted from a pixel location is dependent only on the R, G, B triplet for that pixel and independent of the driving signals for other pixels, of the position of the pixel on the display and of time.

With these assumptions, the operation of the display may be modeled mathematically as a radiance spectrum

$$
f(\lambda ; \mathrm{R}, \mathrm{G}, \mathrm{B})
$$

for each R, G, B triplet, where $\lambda$ denotes the wavelength. For color measurement and control, the visible region of the electromagnetic spectrum covering a wavelength range roughly from $\lambda_{\min }=400 \mathrm{~nm}$ to $\lambda_{\max }=700 \mathrm{~nm}$ is of interest. The color corresponding to the radiance spectrum can then be measured and specified using the CIE system [15], wherein color corresponding to the radiance spectrum is specified by a "tristimulus" vector $\mathbf{t}(\mathrm{R}, \mathrm{G}, \mathrm{B})=\left[t_{1}, t_{2}, t_{3}\right]^{T}$ of the $X Y Z$ tristimulus coordinates ${ }^{1}$ given by

$$
t_{i}=\int_{\lambda_{\min }}^{\lambda_{\max }} c_{i}(\lambda) f(\lambda ; \mathrm{R}, \mathrm{G}, \mathrm{B}) d \lambda
$$

where $t_{1}, t_{2}, t_{3}$ are, respectively, the CIE $X, Y$, and $Z$ tristimulus values and $c_{1}(\lambda), c_{2}(\lambda), c_{3}(\lambda)$ are, respectively, the CIE $2^{\circ}$ color-matching functions [15] $\bar{x}(\lambda), \bar{y}(\lambda), \bar{z}(\lambda)$. The CIE $X Y Z$ tristimulus values may be transformed into a number of different color spaces as required [16] and, conversely, values from different color spaces may be transformed into CIE $X Y Z$ tristimulus values.

The knowledge of $\mathbf{t}(\mathrm{R}, \mathrm{G}, \mathrm{B})$, for all values of RGB signals, constitutes a "forward model" for the display, which provides the color tristimulus values for a pixel given the RGB signal values. In practice, the goal of color calibration is to allow accurate display of images whose pixel colors are specified in terms of their CIE $X Y Z$ tristimulus values (or equivalent color coordinates in alternate color spaces). Thus, in order to utilize the color characterization in practice, we require the inverse of the "forward model," which provides a mapping from each desired color in CIE $X Y Z$ tristimulus space to the corresponding R, G, B triplet for the display. For the rather general model described above, this inverse needs to be determined by numerically inverting the multidimensional forward model. However, as will be apparent from the following discussion, with suitable simplifying assumptions the inverse may also be determined much more readily.

The generic mathematical model of a display as a radiance distribution for each driving RGB triplet value is complex and requires a large number of measurements for color char-

\footnotetext{
${ }^{1}$ Familiarity with basics of color is assumed here, for a recent tutorial review of colorimetry and a discussion of the state of the art in color imaging, the reader is referred to [16] and [17].
}

acterization. The design and operating physics of displays often ensures that the red, green, and blue channels function independently of each other and this assumption of channel independence is commonly employed in color characterization models. Mathematically, the channel-independence assumption implies that the radiance spectrum corresponding to an RGB triplet can be separated into functions dependent only on the individual $\mathrm{R}, \mathrm{G}$, and $\mathrm{B}$ values, i.e.,

$$
f(\lambda ; \mathrm{R}, \mathrm{G}, \mathrm{B},)=f_{\mathrm{r}}(\lambda, \mathrm{R})+f_{\mathrm{g}}(\lambda, \mathrm{G})+f_{\mathrm{b}}(\lambda, \mathrm{B})+f_{0}(\lambda)
$$

where $f_{\mathrm{r}}(\lambda, \mathrm{R})$ represents the light produced by the red channel in response to the input value $\mathrm{R}$ and, in a similar fashion, $f_{\mathrm{g}}(\lambda, \mathrm{G})$ and $f_{\mathrm{b}}(\lambda, \mathrm{B})$ represent the light from the green and blue channels, respectively, and $f_{0}(\lambda)$ accounts for light reflected by the display (flare) and/or light emitted from a dark pixel (with $\mathrm{R}=0, \mathrm{G}=0$, and $\mathrm{B}=0$ ).

The channel-independence assumption allows the complete characterization of the display based on a characterization of the individual RGB channels. It, therefore, reduces the problem of color characterization of the display from a threedimensional (3-D) characterization into three much simpler one-dimensional (1-D) characterizations. The problem can be simplified further using the additional assumption that the spectrum of light from a channel has the same basic shape and only undergoes a scaling in amplitude as the driving signal for that channel is varied. Mathematically, this assumption can be stated for the red channel as

$$
f_{\mathrm{r}}(\lambda, \mathrm{R})=v_{\mathrm{r}}(\mathrm{R}) s_{\mathrm{r}}(\lambda)
$$

where $s_{\mathrm{r}}(\lambda)$ represents the radiance spectra of the light from the red channel at the maximum value of the red driving signal and $v_{\mathrm{r}}(\mathrm{R})$ denotes the amplitude scaling factor. Note that by virtue of our definition, $0 \leq v_{\mathrm{r}}(\mathrm{R}) \leq 1$. For the green and blue channels, the assumption results in similar decompositions: $f_{\mathrm{g}}(\lambda, \mathrm{G})=v_{\mathrm{g}}(\mathrm{G}) s_{\mathrm{g}}(\lambda)$ and $f_{\mathrm{b}}(\lambda, \mathrm{B})=v_{\mathrm{b}}(\mathrm{B}) s_{\mathrm{b}}(\lambda)$, where the terms are defined analogously to those for the red channel. The functions $v_{\mathrm{r}}(\cdot), v_{\mathrm{g}}(\cdot)$, and $v_{\mathrm{b}}(\cdot)$ are commonly referred to as the tone-response curves (TRCs) of the red, green, and blue channels. Since they correspond to the amplitude scaling factors for the light spectrum output of each of the channels with increasing driving signals, they are typically monotonically increasing functions. Note that due to the implicit normalization above, the TRCs are unity at the maximum value of the driving signal for the corresponding channel.

From the linearity of (2), it follows that the assumptions of (3) and (4) can alternately be written in terms of the tristimulus values instead of spectra as

$$
\begin{aligned}
\mathbf{t}(\mathrm{R}, \mathrm{G}, \mathrm{B}) & =\mathbf{t}_{\mathrm{r}}(\mathrm{R})+\mathbf{t}_{\mathrm{g}}(\mathrm{G})+\mathbf{t}_{\mathrm{b}}(\mathrm{B})+\mathbf{t}_{0} \\
\mathbf{t}_{\mathrm{r}}(\mathrm{R}) & =v_{\mathrm{r}}(\mathrm{R}) \mathbf{t}_{\mathrm{mr}} \\
\mathbf{t}_{\mathrm{g}}(\mathrm{G}) & =v_{\mathrm{g}}(\mathrm{G}) \mathbf{t}_{\mathrm{mg}} \\
\mathbf{t}_{\mathrm{b}}(\mathrm{B}) & =v_{\mathrm{b}}(\mathrm{B}) \mathbf{t}_{\mathrm{mb}}
\end{aligned}
$$

where the bold face tristimulus terms in (5) represent the tristimulus values corresponding to the spectral terms defined earlier and $\mathbf{t}_{\mathrm{mr}}, \mathbf{t}_{\mathrm{mg}}$, and $\mathbf{t}_{\mathrm{mb}}$ represent the tristimulus 


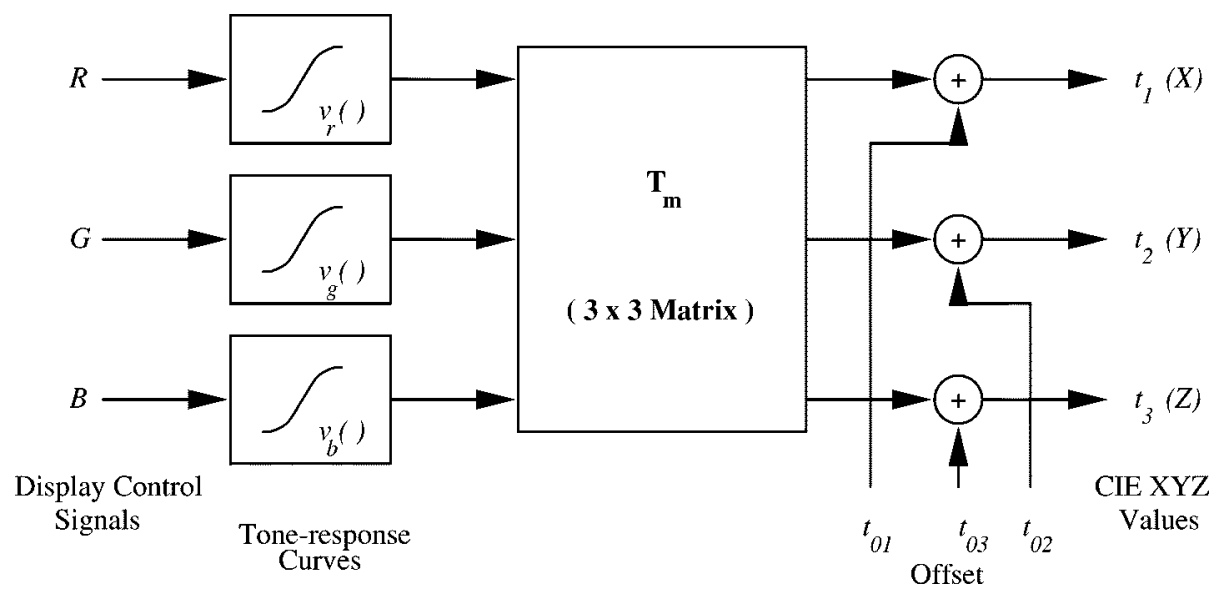

Fig. 1. Graphical representation of the display forward model.

values at the maximum driving signal for the red, green, and blue channels, respectively. The complete model of (5)-(8) is shown in Fig. 1.

Note that (6)-(8) imply that the tristimulus contribution from a channel is simply a scalar multiple of its tristimulus value at the maximum driving signal. For two-dimensional (2-D) graphical representation of colors, it is common to employ chromaticity coordinates that represent a normalization of the 3-D tristimulus values. In particular, the CIE $x, y$ chromaticity coordinates [15], [16], [18] corresponding to a color with CIE $X Y Z$ tristimulus vector $[X Y Z]^{T}$ are defined as

$$
\begin{aligned}
& x=\frac{X}{X+Y+Z} \\
& y=\frac{Y}{X+Y+Z} .
\end{aligned}
$$

Since chromaticity values represent a normalization of the tristimulus values that is invariant to scalar multiplication, (6)-(8) are equivalent to the assumption that the chromaticity of a channel remains constant independent of the value of the driving signal for that channel. This is referred to as the "channel-chromaticity-constancy assumption." Since (4) is the physical basis for the channel-chromaticity constancy, by extension the "channel-chromaticity-constancy assumption" will also be used to refer to (4).

Equations (5)-(8) can be combined as

$$
\begin{aligned}
\mathbf{t}(\mathrm{R}, \mathrm{G}, \mathrm{B})= & {\left[\mathbf{t}_{\mathrm{mr}}, \mathbf{t}_{\mathrm{mg}}, \mathbf{t}_{\mathrm{mb}}\right] } \\
& \cdot\left[v_{\mathrm{r}}(\mathrm{R}), v_{\mathrm{g}}(\mathrm{G}), v_{\mathrm{b}}(\mathrm{B})\right]^{T}+\mathbf{t}_{0} \\
= & \mathbf{T}_{m}\left[v_{\mathrm{r}}(\mathrm{R}), v_{\mathrm{g}}(\mathrm{G}), v_{\mathrm{b}}(\mathrm{B})\right]^{T}+\mathbf{t}_{0}
\end{aligned}
$$

where $\mathbf{T}_{m} \equiv\left[\mathbf{t}_{\mathrm{mr}}, \mathbf{t}_{\mathrm{mg}}, \mathbf{t}_{\mathrm{mb}}\right]$ is the matrix with the maximum amplitude tristimuli for the RGB channels as its columns.

As mentioned earlier, practical use of the display color calibration requires the inverse of the device characterization model, which provides the RGB values corresponding to a desired tristimulus value. The model of (12) can be readily inverted to obtain the RGB values corresponding to a desired tristimulus value $\mathbf{t}$ as

$$
\begin{aligned}
{[\mathrm{R}, \mathrm{G}, \mathrm{B},] } & =\left[v_{\mathrm{r}}^{-1}\left(\mathrm{R}^{\prime}\right), v_{\mathrm{g}}^{-1}\left(\mathrm{G}^{\prime}\right), v_{\mathrm{b}}^{-1}\left(\mathrm{~B}^{\prime}\right)\right] \\
{\left[\mathrm{R}^{\prime}, \mathrm{G}^{\prime}, \mathrm{B}^{\prime}\right] } & =\mathbf{T}_{m}^{-1}\left(\mathbf{t}-\mathbf{t}_{0}\right)
\end{aligned}
$$

The above equations are based on the assumptions that: 1) the matrix $\mathbf{T}_{m}$ is nonsingular and 2) that the TRCs $v_{\mathrm{r}}(\cdot)$, $v_{\mathrm{g}}(\cdot)$, and $v_{\mathrm{b}}(\cdot)$ are invertible functions. Typical displays are designed so that their channels are colorimetrically independent [16], which ensures that $\mathbf{T}_{m}$ is nonsingular, and the monotonic nature of the TRCs ensures invertibility.

From the above discussion, it is apparent that for the simplified model of (5)-(8), the inverse model is readily computed using the inverse of the 1-D TRCs for each of the channels and the inverse of the $3 \times 3$ matrix $\mathbf{T}_{m}$. This is significantly simpler than direct numerical computation of the 3-D forward model for the display. The matrix inverse and the inverse of the TRCs may be precomputed and the latter may be computationally implemented as a 1-D lookup table (LUT). Fig. 2 illustrates this inverse model, depicting the process of converting from CIE $X Y Z$ to display RGB using an offset-correction $3 \times 3$ matrix and inverse tone-response corrections. In order to display a calibrated image on the display, these operations would need to be applied to each pixel as the color-correction step. The inverse model of (13) and (14) is fairly computationally efficient and can be directly used for mapping of images into display RGB color coordinates prior to display. As opposed to this, the more general models of (1) and (3) require intensive computation for the determination of the inverse. The runtime mapping of images to display coordinates for these general models relies on memory intensive 3-D LUTs that store the inverse model.

\section{CRT COLOR CALIBRATION}

The models of Section II have been successfully applied to the characterization of CRT monitors since the early 1980s [4]. The assumptions of channel independence and channelchromaticity constancy described in the previous section are 


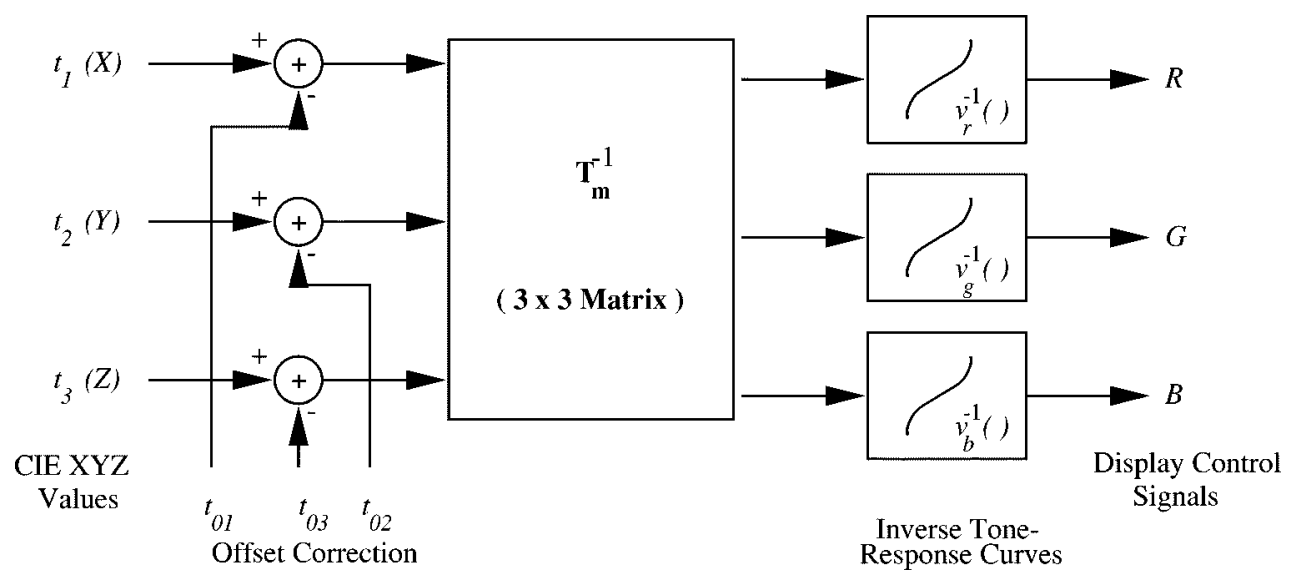

Fig. 2. Graphical representation of the display inverse model.

specifically referred to as "gun independence" and "phosphor constancy" in the context of CRT monitors. The physics of CRTs strongly supports these assumptions and they have also been extensively validated in experiments [5], [9].

For CRTs, the final model of Section II, can be further simplified by using a parametric mathematical model for the TRCs for the individual channels that is derived from the power-law relation between grid voltage and beam current for a vacuum tube [19], [20]. The expression for the redchannel TRC resulting from the power-law relation can be written as [8], [21]

$$
v_{\mathrm{r}}(\mathrm{R})=\left(\left(1-\beta_{\mathrm{r}}\right) \frac{\mathrm{R}}{\mathrm{R}_{\max }}+\beta_{\mathrm{r}}\right)^{\gamma_{\mathrm{r}}}
$$

where $\mathrm{R}_{\max }$ corresponds to the maximum value for the redchannel signal and $\beta_{\mathrm{r}}$ and $\gamma_{\mathrm{r}}$ represent the offset and exponent parameters of the model. Analogous expressions apply for the green and blue channels.

For appropriate setup of the monitor offset and brightness controls [8], the offset term $\beta_{\mathrm{r}}=0$ and the relation simplifies to

$$
v_{\mathrm{r}}(\mathrm{R})=\left(\frac{\mathrm{R}}{\mathrm{R}_{\max }}\right)^{\gamma_{\mathrm{r}}}
$$

which is the commonly used power-law relation for CRTs [22, p. 107]. Similar relations can be obtained for the blue and green channels with corresponding exponents $\gamma_{\mathrm{g}}$ and $\gamma_{\mathrm{b}}$, respectively, in the power-law relation. The exponents $\gamma_{\mathrm{r}}, \gamma_{\mathrm{g}}$, and $\gamma_{\mathrm{b}}$ are typically equal and their value is commonly referred to as the "gamma" of the CRT. The numerical value of gamma for a CRT is typically around 2.2, though the effective "gamma" seen by an application may be influenced by the display and operating system settings [23].

With the parametric form of (16) for the TRCs, (13) reduces to

$$
\begin{aligned}
& {[\mathrm{R}, \mathrm{G}, \mathrm{B},]} \\
& \quad=\left[\left(\mathrm{R}^{\prime}\right)^{1 / \gamma_{\mathrm{r}}} \mathrm{R}_{\max },\left(\mathrm{G}^{\prime}\right)^{1 / \gamma_{\mathrm{g}}} \mathrm{G}_{\max },\left(\mathrm{B}^{\prime}\right)^{1 / \gamma_{\mathrm{b}}} \mathrm{B}_{\max }\right]
\end{aligned}
$$

which is commonly referred to as gamma correction. It is worth mentioning that uniform quantization of gamma-corrected signals results in wider quantization intervals at higher amplitudes where the sensitivity of the eye is also lower. Therefore, just like speech companding, gamma correction of color tristimuli prior to quantization in a digital system (or transmission in a limited bandwidth system) reduces the perceptibility of errors and contours in comparison to a scheme in which no gamma correction is used [24]-[27], [28, p. 393].

Most present-day CRT monitors are manufactured using the same set of red, green, and blue phosphors and the power-law relation is a fundamental characteristic of vacuum tubes. CRTs, therefore, tend to be fairly close to each other in their basic color characteristics. Because of the extremely widespread use of CRTs, it is common for images to be stored and transmitted in a using a color representation that is suitable for direct display on a CRT. Recently, the sRGB color-space [29] has been defined to bless and crystallize this de facto standard and to provide extensions that allow for incorporation of additional information on the viewing conditions, which can have a significant impact on human perception of displayed images.

\section{AMLCD DisPlay PHYSICS AND COLOR CHARACTERISTICS}

The most common LCDs for computers are backlit AMLCDs of the "twisted nematic" type [30, p. 72]. These are manufactured by deposition and patterning of (active) pixel electronics on a glass substrate. Each pixel element consists of a pair of linear polarizers with liquid-crystal (LC) material sandwiched in between. Fig. 3 illustrates a pixel element. The two linear polarizers are orthogonally oriented; light does not pass through the display except for actions of the LCs. The surfaces adjacent to the LC molecules are typically designed so that (in the absence of any electric field) the LC molecules align in a $90^{\circ}$ twisted configuration, which rotates the plane of polarization of incident linearly polarized light by a $90^{\circ}$ angle [31, pp. 429-430]. The "input" polarizer on the backside polarizes the light coming from the lamp behind the display. This polarized light encounters the LC molecules, which rotate its plane of polarization by $90^{\circ}$, allowing it to pass through the output polarizer, resulting in an ON pixel. The pixel is turned off by the application of an electric field. Due to their 


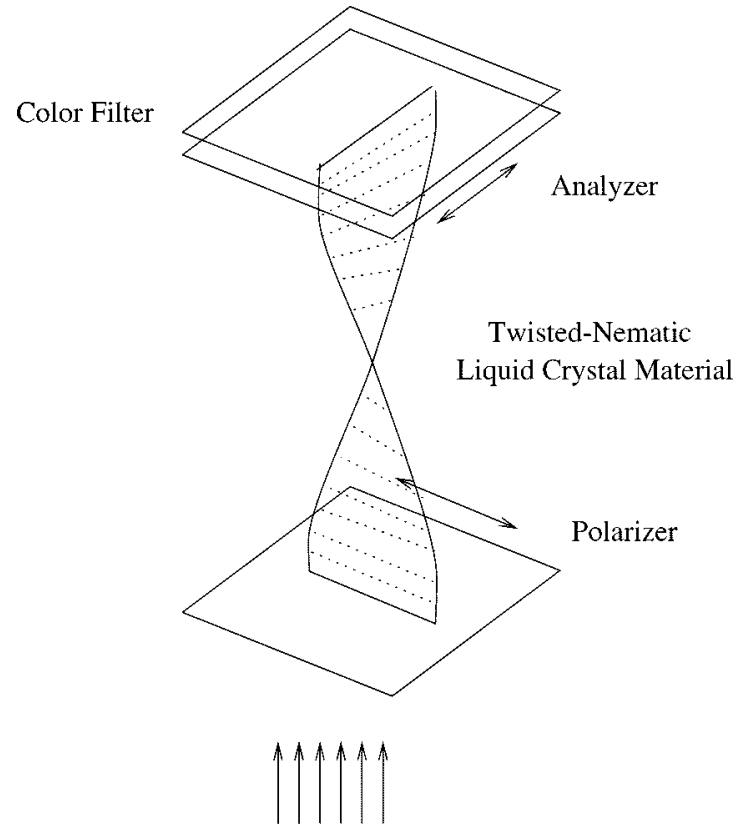

Back Light

Fig. 3. Structure of an LCD pixel.

"dielectric anisotropy," the LC molecules tend to align with the electric field and move away from their twisted state. In a strong-enough electric field, the molecules are almost completely aligned with the electric field. This causes the pixel to be turned off as the $\mathrm{LC}$ molecules no longer produce the $90^{\circ}$ rotation in the plane of polarization that is required for the output polarizer to transmit the light.

Color displays are produced by laying a mosaic of red, green, and blue colored filters on the substrate glass aligned with the pixel array. Quite often, the individual RGB pixels are rectangular and arranged so that three horizontally adjacent rectangular RGB pixels constitute a single square "color pixel" (which is set further apart from other "color pixels" in comparison to the spacing between the individual RGB pixels). The display, thus, appears to be composed of stripes of rectangular RGB pixels going vertically across the screen. The backlight is typically a fluorescent lamp with three prominent peaks in the red, green, and blue regions of the spectrum.

In most AMLCD color displays, the RGB pixels are driven and controlled independently. The emitted light is combined and averaged in the eye (just as for CRTs). Therefore, the three RGB channels combine through simple addition of light and one can expect channel independence to hold for these displays. The model of (3) is, therefore, suitable for color characterization. As indicated earlier, this allows a complete characterization of the display from a per-channel characterization. Furthermore, if the switching mechanism of the LCD pixel cell described above is spectrally nonselective, i.e., when a pixel is driven by applying a voltage the percent change in spectral transmittance is the same across all wavelengths, the channel-chromaticity-constancy assumption of (4) also applies, further simplifying the characterization. The TRCs $v_{\mathrm{r}}(\cdot), v_{\mathrm{g}}(\cdot)$, and $v_{\mathrm{b}}(\cdot)$ then correspond to what is commonly referred to as the electrooptic response in LCD terminology. The electrooptic response of an LCD pixel cell (for on-axis viewing) tends to be an S-shaped curve (like sigmoidal functions) [30]. This response is quite different from the power-law relation for CRTs and cannot readily be modeled by the parametric power-law relation of (15).

Since LCDs were first deployed in an environment dominated by CRTs, the backlights and color filters for these devices were designed to produce red, green, and blue channel chromaticities identical to the common CRT phosphor chromaticities. This allows the devices to be directly driven by the same signals as those used for CRTs with only 1-D compensations for the per-channel TRCs. Often displays incorporate builtin/switchable per-channel compensation curves, which could change the actual observed TRCs for the display. These compensation curves are also typically set up to effectively mimic a power-law relation [10] between the driving signals and the observed luminance.

\section{EXPERIMENTAL PROCEDURE}

A prototype LCD [13] was chosen for experimental study in order to evaluate the applicability of the color-calibration models of Section II to LCDs. A commercially available CRT monitor was also studied in parallel to provide comparative data. Both displays were allowed to warm up for over 45 min to reduce the effects of any transient variations upon poweron. For the purposes of color characterization and evaluation, on either display, a number of spatially uniform color patches were displayed in the central region and measured using a PR705 spectroradiometer that provides full spectral radiance (in W/sr/ $/ \mathrm{m}^{2}$ units) for each of the patches at a 2- nm sampling resolution in the range $380-780 \mathrm{~nm}$ (as opposed to a colorimeter that provides only CIE $X Y Z$ or equivalent values). The patches were partitioned a priori into a characterization set used for performing the color calibration and an independent test set for evaluating the accuracy of the characterization. All measurements were made at a $0^{\circ}$ viewing angle (with respect to the normal to the screen). The measurements for the patches were made in a dark room with minimal stray light. Flare (reflection from the display screen) for normal viewing conditions was measured independently for each of the displays with typical room lighting turned on. The display background around the patches displayed was set to black in order to avoid any stray light in the measurements and to avoid overloading of the CRT driving circuits. The CRT color temperature setting for the white point was selected as $6500 \mathrm{~K}$ from the available options. All measurements were made with a $1^{\circ}$ measuring aperture setting on the PR705 spectroradiometer.

Both displays were driven by 24-bit display adapters with 8 bits each for the red, green, and blue channels. The characterization patches consisted of ramps with 33 levels each for each of the R, G, B channels (e.g., the red-channel characterization patches consisted of patches with $\mathrm{G}=\mathrm{B}=0$ and $R$ values uniformly sampling the range from 0 to $R_{\max }=$ 


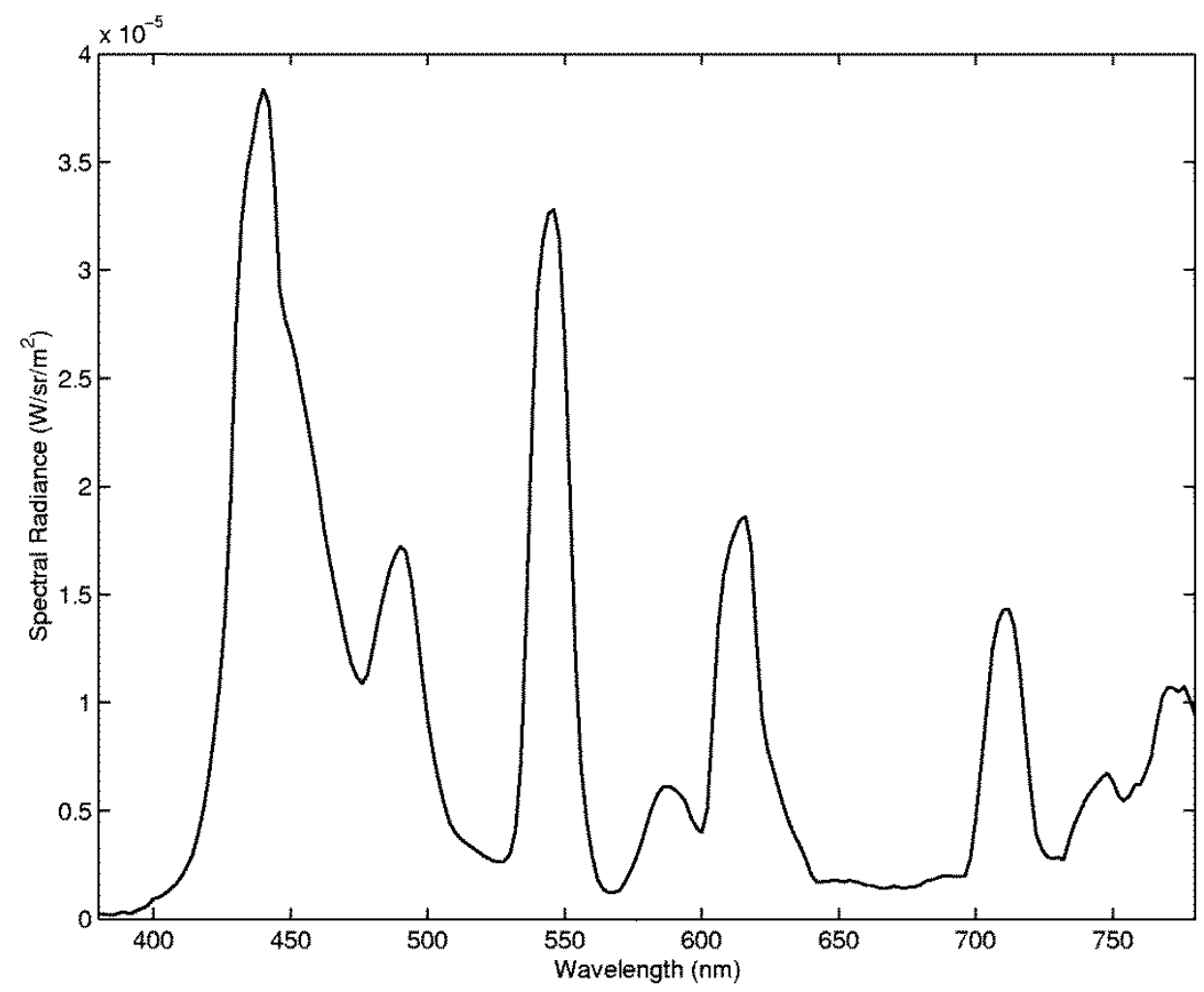

Fig. 4. Spectral radiance of $\mathrm{LCD}$ black $(\mathrm{R}=\mathrm{G}=\mathrm{B}=0)$.

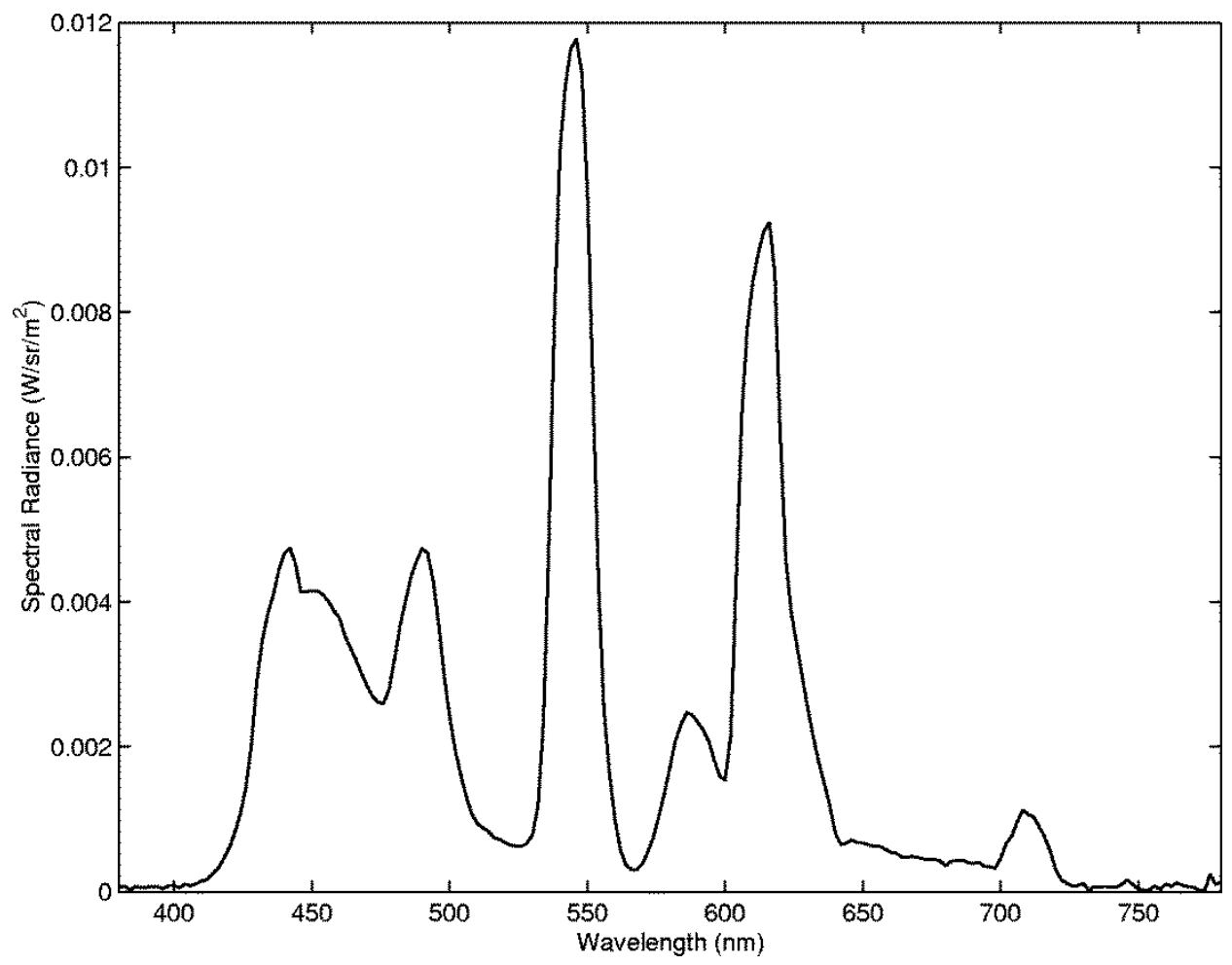

Fig. 5. Spectral radiance of $L C D$ white $(R=G=B=255)$.

255). The test set consisted of 64 independent test patches representing a $4 \times 4 \times 4$ uniform sampling of the RGB cube. Where required, color in CIEXY Z/CIELAB coordinates [15] was computed from the spectral measurements as in (2).

\section{SPECTRAL CHARACTERIZATION}

The spectra corresponding to display black $(\mathrm{R}=\mathrm{G}=$ $\mathrm{B}=0)$ and white $(\mathrm{R}=\mathrm{G}=\mathrm{B}=255)$ are shown in Figs. 4 and 5, respectively, for the LCD. The white patch 


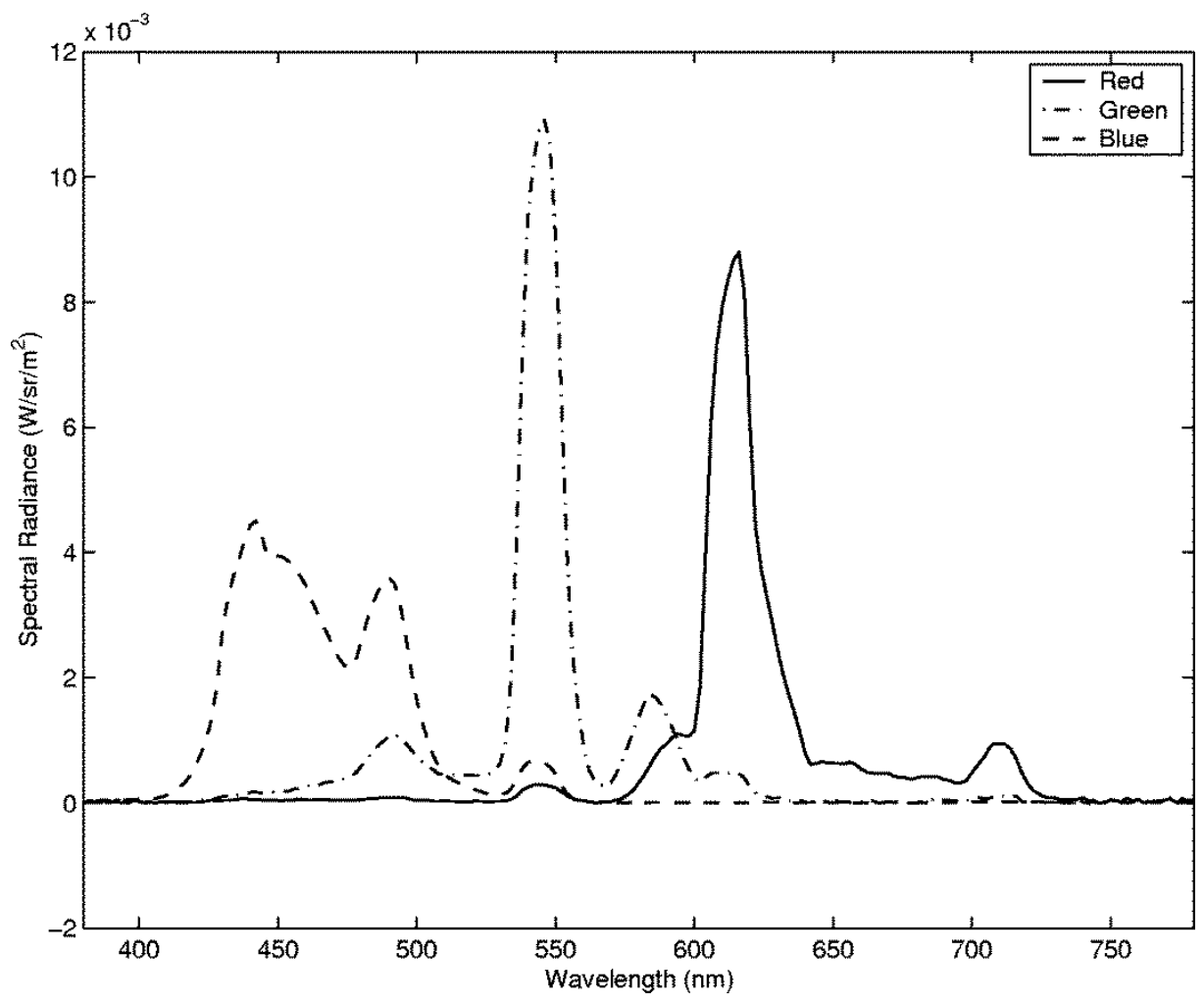

Fig. 6. Spectral radiance of LCD red, green, and blue channels at maximum amplitude.

corresponds to all the RGB pixels turned on and this spectrum represents the average of the spectra obtained when the backlight is filtered through the mosaic of RGB filters. Note that the black spectrum has a much lower absolute value than the white (the ratio of luminance of black to white is approximately $1: 357$ ), but the shape of the black spectrum is similar to that of the white. This suggests that the pixels (and the region between the pixels) transmit a small fraction of the backlight even in the OFF state. This residual light from the display is a constant "additive offset," which is present in all measurements (just like flare). The additive offset is readily accounted for in the model of (3) by simply incorporating it in the term $f_{0}(\lambda)$ (along with any flare). Assuming channel independence, the individual RGB channel spectral terms in then model of (3) are then obtained by subtracting this offset from the spectral measurements for the red, green, and blue ramps constituting the characterization set.

Fig. 6 shows the (offset-corrected) spectra for the red, green, and blue display channels at the maximum driving signal (i.e., a digital value of 255) for each of the channels. If, in addition to channel-independence the constant-channel-chromaticity assumption of (4) is also assumed to hold, the TRCs of the red, green, and blue channels can also be computed from the measurements for the individual channel ramps using least-squares. This process is illustrated for the red channel as

$$
v_{\mathrm{r}}(\mathrm{R})=\frac{\sum_{\lambda}\left(f(\lambda ; \mathrm{R}) s_{\mathrm{r}}(\lambda)\right)}{\left(\sum_{\lambda} s_{\mathrm{r}}^{2}(\lambda)\right)^{1 / 2}} .
$$

In order to partly test the validity of the model assumptions for LCDs, residual spectral differences were computed between the measurements and the model of (4) using the leastsquares approximation of (18). The residual errors are rather small in comparison to the measurements themselves with spectral mean-squared errors (SMSEs) of $-37.65,-36.25$, and $-29.57 \mathrm{~dB}$, respectively, for the red, green, and blue channels, where the SMSE for the red channel is defined as

$\operatorname{SMSE}(\mathrm{dB})$

$$
=10 \log _{10}\left(\frac{\sum_{\lambda} \sum_{\mathrm{R}}\left(f(\lambda ; \mathrm{R})-v_{\mathrm{r}}(\mathrm{R}) s_{\mathrm{r}}(\lambda)\right)^{2}}{\sum_{\lambda} \sum_{\mathrm{R}} f^{2}(\lambda ; \mathrm{R})}\right)
$$

and the SMSE for the other channels is similarly defined. The model of (4) is, therefore, a fairly accurate model for the individual ramps. Plots of the spectral residuals also do not show any systematic trends except in the blue region of the spectrum for the green and blue ramp residuals. Wavelength dependence of the LCD switching mechanism is one potential cause for these observed systematic deviations.

The TRCs $v_{\mathrm{r}}(\cdot), v_{\mathrm{g}}(\cdot)$, and $v_{\mathrm{b}}(\cdot)$ for the red, green, and blue channels obtained from the above described procedure are shown in Fig. 7. Note that the TRCs have the characteristic S-shape expected from the raw optoelectronic responses for an LCD pixel. Also note that the TRCs for red, green, and blue channels are not identical.

For the purposes of comparison, the data measured from CRT was analyzed using identical procedures. The radiance spectra for black and white are shown in Figs. 8 and 9, respectively, for the CRT. Fig. 10 shows the (offset-corrected) 


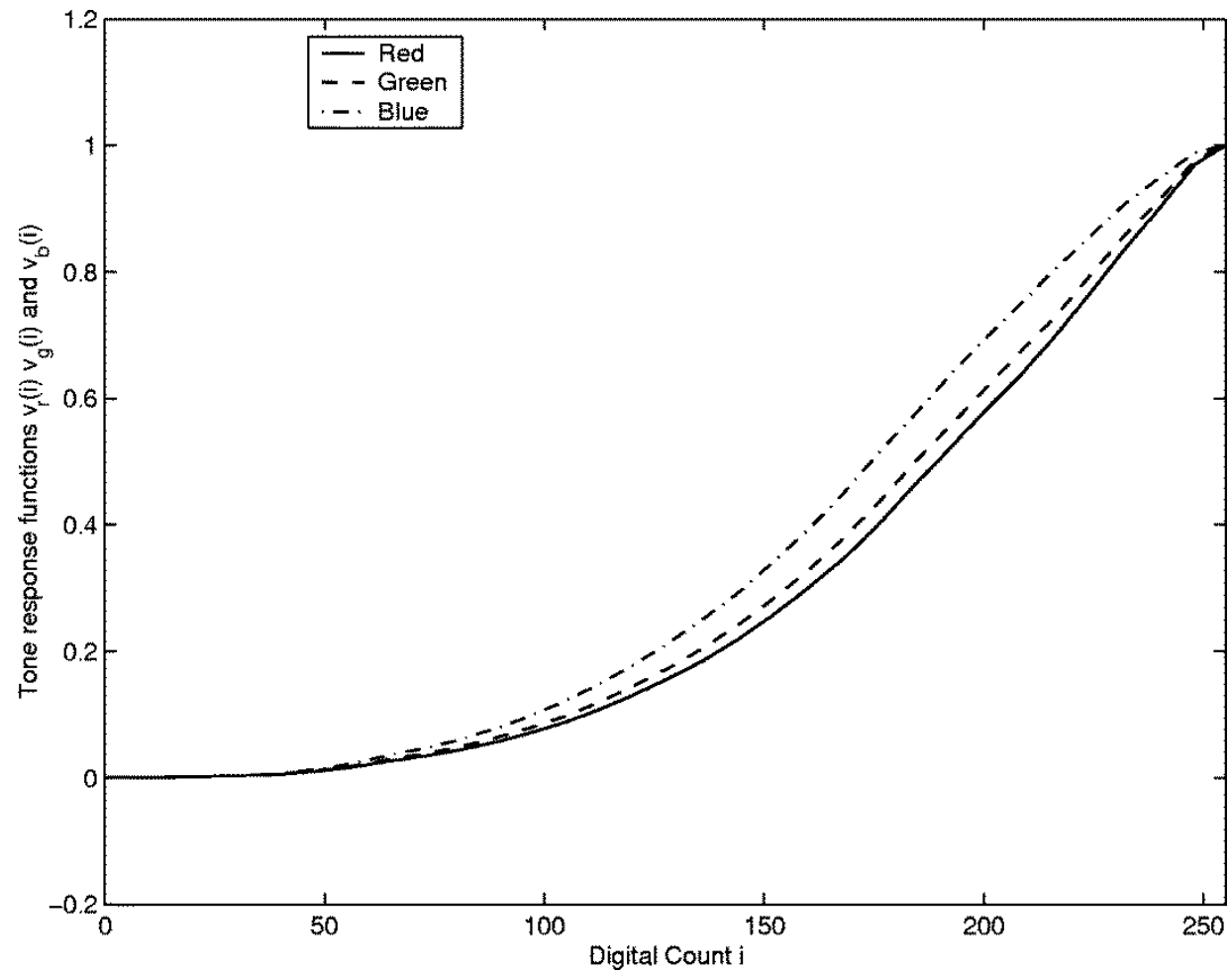

Fig. 7. TRCs for the LCD red, green, and blue channels.

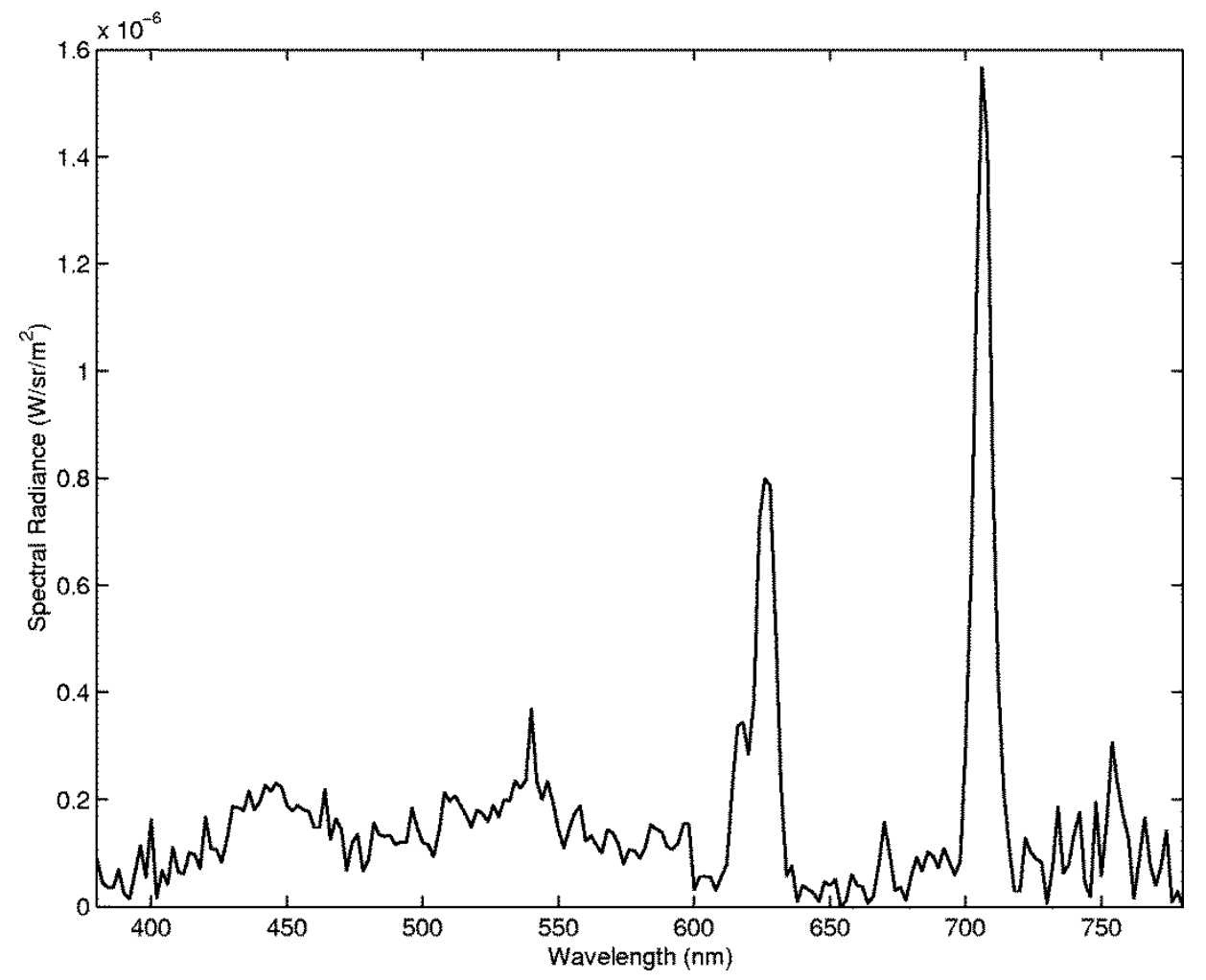

Fig. 8. Spectral radiance of CRT display black $(R=G=B=0)$.

spectra for the CRT red, green, and blue display channels at the maximum driving signal (i.e., 255). Note that the CRTs radiances are significantly lower than the corresponding radiances for the LCD. Also, for the near-ideal setup used for measurements (of a dark room with no flare), the spectrum of the CRT black appears to be primarily measurement noise with a very small contribution from the red phosphors, which is evidenced by the two peaks in the black spectrum at the same locations as the red channel. For these conditions, the black offset may be ignored without significant loss of ac- 


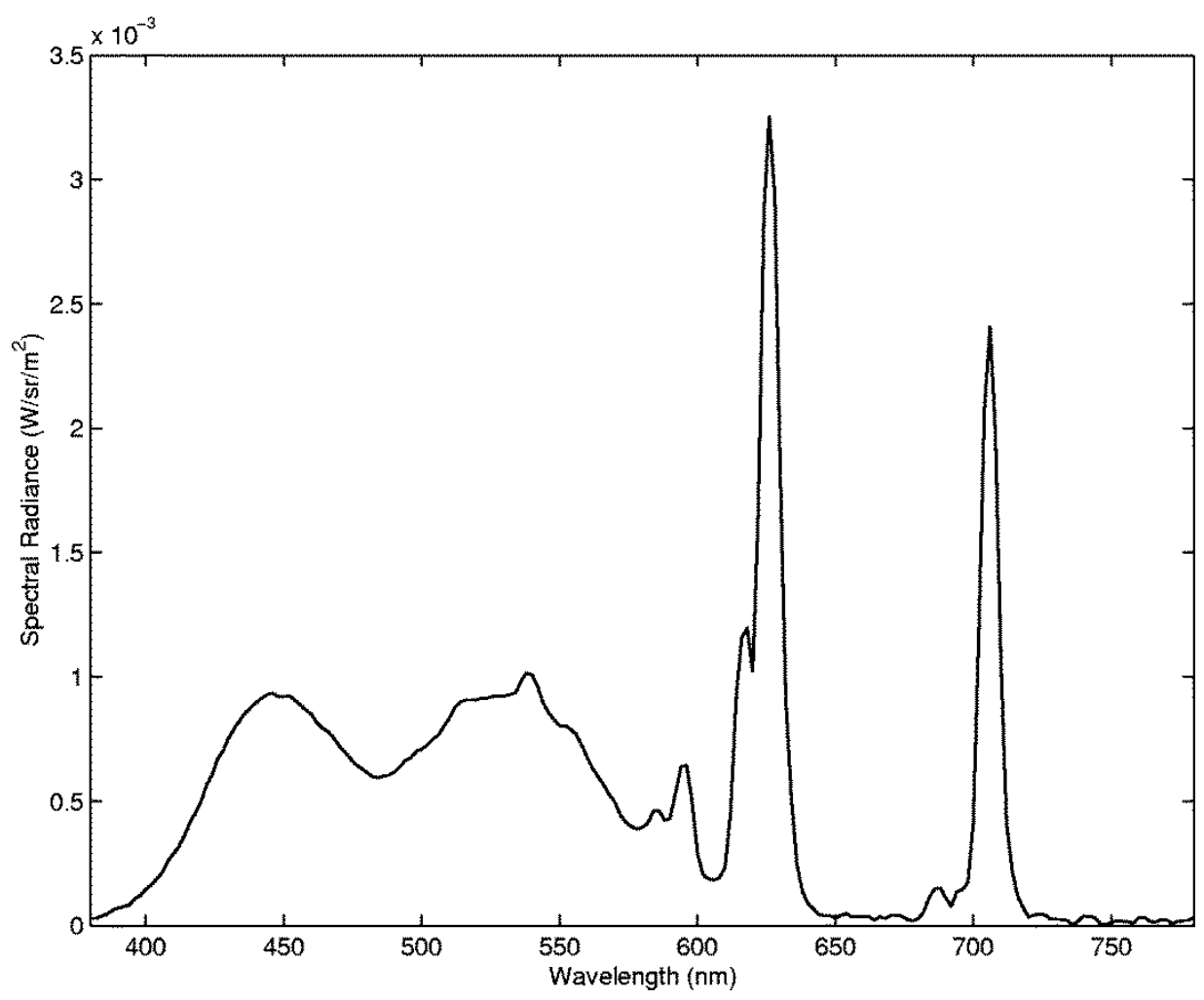

Fig. 9. Spectral radiance of CRT display white $(R=G=B=0)$.

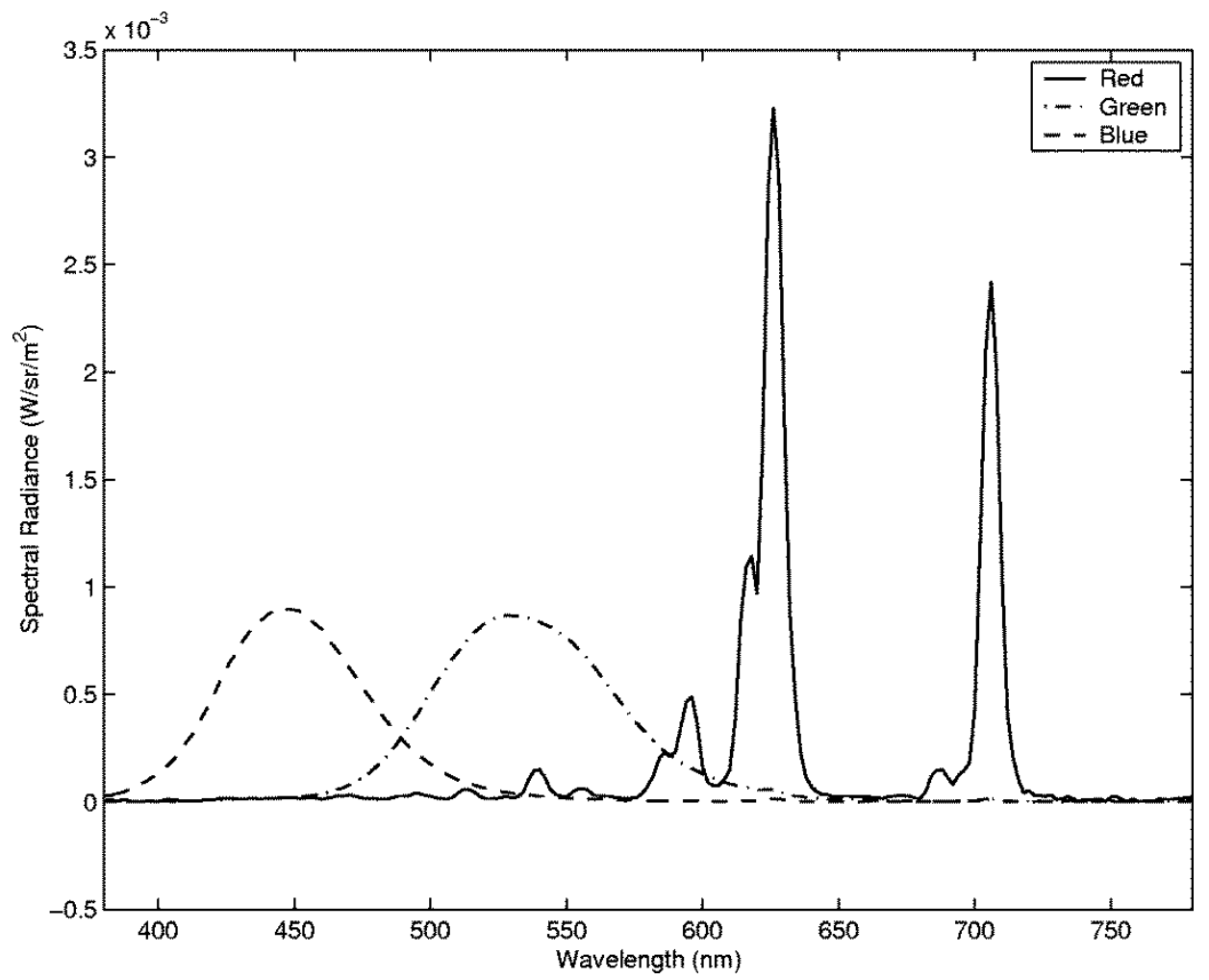

Fig. 10. Spectral radiance of CRT display red, green, and blue channels at maximum amplitude.

curacy, i.e., $f_{0}(\lambda)=0$ can be assumed in the model of (3). Under these ideal measurement conditions, the dynamic range for the CRT, i.e., the ratio of white to black luminances is approximately $4351: 1$. By comparing Figs. 6 and 10, it is clear that the spectral characteristics of LCDs and CRTs differ significantly. The LCD red and green channels have unimodal spectral radiance distributions with fairly narrow spectral widths, whereas the CRT red-channel spectrum has 


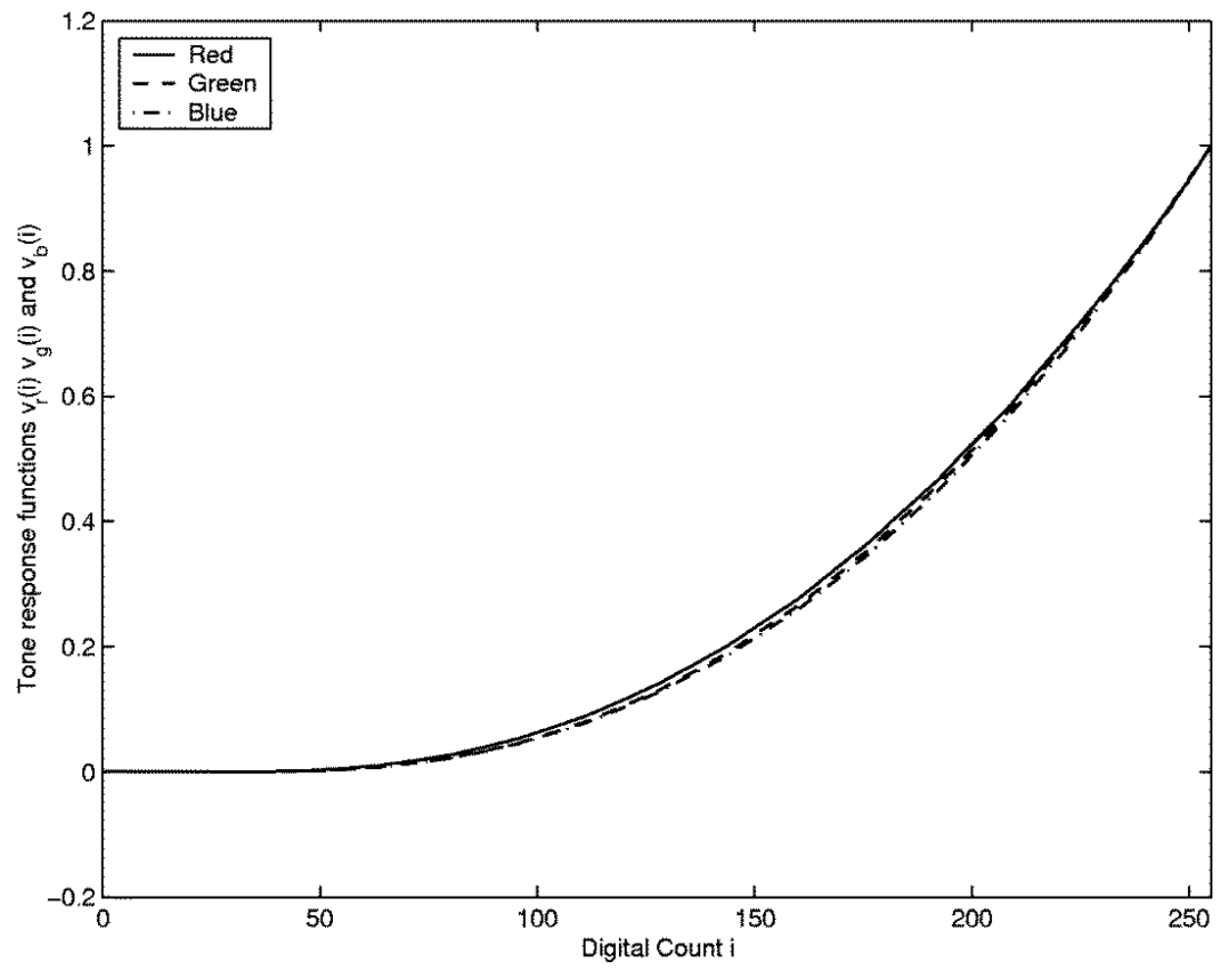

Fig. 11. TRCs for the CRT display red, green, and blue channels.

two narrow peaks and the green- and blue-channel spectra are fairly smooth with relatively large spectral widths.

The model of (4) using the least-squares approximation of (18) for the TRCs was also evaluated for the CRT. In this case, the SMSEs were $-38.30,-43.01$, and $-41.92 \mathrm{~dB}$, respectively, for the red, green, and blue channels. The significantly smaller values of the SMSEs indicates that the model of (4) models the behavior of CRTs to a greater degree of accuracy than LCDs. Plots of the model residual spectra are also devoid of any systematic trends. The CRT TRCs for the red, green, and blue channels are shown in Fig. 11. The TRCs are in agreement with the power-law (gamma) relationship of (15) (the best approximations for the gamma for the red, green, and blue channels were $2.34,2.36$, and 2.43 , respectively). Compared to the LCD TRCs, the TRCs for the CRT RGB channels are fairly close to each other.

With the assumptions of channel-independence and channel-chromaticity constancy, the per-channel spectral characterizations can be used with the model of (3) and (4) to predict the spectral radiance for the display corresponding to any RGB value. For both the LCD and the CRT display, predictions for the 64 independent test patches (representing a $4 \times 4 \times 4$ uniform sampling of the RGB cube) were made using the TRCs determined in (18). These predictions were compared with the actual measurements for the test patches and SMSEs were evaluated. The SMSE for the LCD was $-32.07 \mathrm{~dB}$ and for the CRT display the SMSE was -37.14 $\mathrm{dB}$. Both values are quite small indicating that the model predictions provide close approximations to the measurements. The 5-dB lower value for the CRT SMSE indicates that the CRT measurements for the test patches are in better agreement with the model than the LCD measurements, a trend that was also observed in the per-channel case. Plots of the spectral errors reinforce this observation: while the errors for the CRT appear random, for the LCD the errors are not completely random. The predominant trend is the presence of mostly positive errors around the spectrum locations corresponding to the three predominant peaks in the white-patch spectrum.

\section{COLORIMETRIC CHARACTERIZATION}

The measured characterization and test spectra and the spectral predictions obtained by using the models were converted to CIEXYZ tristimulus values [as indicated in (2)] from which CIELAB values were calculated using the respective display white measurements as the white point. These CIELAB values were then used to compute the color errors in the characterization in $\triangle E_{a b}^{*}$ [15] and $\triangle E_{94}^{*}$ [32] units, which provide better agreement with the perceived magnitude of the color error than SMSE or mean-squared error in tristimulus space [18], [32]. These errors are tabulated in Table 1 for the LCD and in Table 2 for the CRT display. Both tables report the errors over the characterization RGB ramps and the test patches separately and both the average and maximum color errors over each of these data sets are tabulated.

For the CRT display, the color errors from the calibration model are extremely small, with even the maximum color error under $1.0 \triangle E_{94}^{*}$ unit. This indicates that the model of (5)-(8) models the operation of the CRT remarkably well. For the LCD, the average color error over the test set is just around 1.0 $\triangle E_{94}^{*}$ unit and the maximum errors is around 2.0 
Table 1

LCD Color-Calibration Errors

\begin{tabular}{c|c|c|c|c}
\hline \multirow{2}{*}{ Sample Set } & \multicolumn{3}{|c}{ Color-Calibration Errors } \\
\cline { 2 - 5 } & \multicolumn{2}{|c|}{$\triangle E_{a b}^{*}$ units } & \multicolumn{2}{c}{$\triangle E_{94}^{*}$ units } \\
\cline { 2 - 5 } & Mean & Max. & Mean & Max \\
\hline Characterization RGB ramps & 2.01 & 6.94 & 0.87 & 2.76 \\
\hline Test $4 \times 4 \times 4$ RGB cube & 2.32 & 4.49 & 1.09 & 2.07 \\
\hline
\end{tabular}

Table 2

CRT Display Color-Calibration Errors

\begin{tabular}{c|c|c|c|c}
\hline \multirow{2}{*}{ Sample Set } & \multicolumn{2}{|c}{ Color-Calibration Errors } \\
\cline { 2 - 4 } & \multicolumn{2}{c|}{$\Delta E_{a b}^{*}$ units } & \multicolumn{2}{c}{$\triangle E_{94}^{*}$ units } \\
\cline { 2 - 5 } & Mean & Max. & Mean & Max \\
\hline Characterization RGB ramps & 0.18 & 1.11 & 0.09 & 0.42 \\
\hline Test $4 \times 4 \times 4$ RGB cube & 0.40 & 0.89 & 0.23 & 0.68 \\
\hline
\end{tabular}

Table 3

Color-Calibration Errors for LCD and CRT Displays Over Test Samples With a "Gamma-Offset" TRC Model

\begin{tabular}{c|c|c|c|c}
\hline Error over Test Set & \multicolumn{3}{|c}{ Color-Calibration Errors } \\
\cline { 2 - 5 }$(4 \times 4 \times 4$ RGB cube $)$ & $\triangle E_{a b}^{*}$ units & \multicolumn{2}{c}{$\triangle E_{94}^{*}$ units } \\
\cline { 2 - 5 } & Mean & Max. & Mean & Max \\
\hline LCD display & 4.95 & 12.30 & 2.44 & 7.66 \\
\hline CRT display & 0.71 & 1.84 & 0.42 & 1.60 \\
\hline
\end{tabular}

$\triangle E_{94}^{*}$. This level of error is acceptable for most imaging applications and therefore the forward model of Fig. 1 and the inverse model of Fig. 2 can be applied for the color calibration of LCDs, for all but the most critical imaging applications.

Since several commercial image processing packages allow for easy "gamma correction" of images, it is also worth determining how closely the power-law-based model of (15) represents the display TRCs. For both the LCD and the CRT, the best "gamma" exponent and offset values for each of the channels were determined through a least-squares fit of the TRCs to (15). The power-law TRCs corresponding to the estimated gamma and offset values from (15) were then used in the forward model of (5)-(8) to obtain predicted tristimulus values for the test samples and color errors were computed as before. The resulting mean and maximum color errors in $\triangle E_{a b}^{*}$ and $\triangle E_{94}^{*}$ units for the LCD and the CRT display are reported in Table 3. Note that the calibration errors for the CRT are only moderately higher than the corresponding ones in Table 2. However, the errors for the LCD are much larger than those in Table 1. Thus, the parametric model of (15) is clearly unsuitable for representing the TRCs of LCDs and color-calibration techniques that rely on the parametric model for display calibration [33]-[35] should not be employed for LCDs.

The errors for the LCD characterization in Table 1 were obtained based on the model of (5)-(8), which makes two fundamental assumptions: 1) channel independence, i.e., the display RGB channels combine in a purely additive fashion, and 2) channel-chromaticity constancy, i.e., the LCD switching mechanism is wavelength independent (nondispersive). Since most LC materials exhibit some dispersion, the second assumption is the weaker of the two assumptions. Furthermore, it is possible to drop this assumption from the characterization process without requiring additional characterization measurements (other than the R, G, B ramps) by using the model of (3) directly. The model can be more conveniently implemented by considering the corresponding version in terms of tristimulus values. CIE $X Y Z$ tristimulus values for each of the RGB channels can be obtained from the measured values for the corresponding ramps by interpolation of each tristimulus value and the channel-independence assumption can then be used to predict the CIEXYZ tristimulus values for any arbitrary combination of RGB values. Using this method, a "forward model" for representing the operation of the LCD can be obtained, as shown in Fig. 12. The model has nine independent interpolation TRCs, three each corresponding to the $X Y Z$ values for each of the three RGB channels. Note that the model can also be readily extended to a spectral one in a similar fashion.

The colorimetric errors obtained for the LCD with this alternate "forward" model are given in Table 4. Note that since the model interpolates the $X Y Z$ measurements obtained from the characterization samples, the error over the characterization set is negligible. The error over the test samples is also reduced in comparison to Table 1. However, the errors are still significantly larger than those for the CRT. Note that while this alternate method of characterization requires no additional measurements, it cannot be directly inverted to obtain an inverse model. Instead, the inversion must be done numerically and the transformation from CIEXYZ to device RGB requires a complete 3-D LUT instead of the simple offset, matrix, and tone-response correction of Fig. 2.

\section{ANGULAR DEPENDENCE OF THE LCD DisPlay}

A common problem with most AMLCDs is one of limited viewing angle. Due to the birefringent nature of LC molecules, polarized light entering an LC material off axis is treated differently from polarized light entering along the optical axis $\left(0^{\circ}\right.$ angle of incidence with respect to the normal). The LC molecules are less effective in performing the proper rotation for polarized light that enters the LC material off axis. The electrooptic transfer function of LCDs therefore tends to be angle dependent [31, p. 430]. In addition the LCs are dispersive and operate differently on different wavelengths of light, especially when responding to offaxis incident light. As one moves off axis (either up or down or from side to side), significant variations in hue and contrast occur with the change in viewing angle [36]. As one moves far enough off axis, the contrast is diminished to the point that it limits the useful viewing angle. Several innovative techniques have been developed to decrease the viewing-angle dependence of LCDs [37]-[40], but the limited useful viewing angle continues to be a significant limitation of LCDs and further improvements in this area are expected to continue.

In order to quantify the level of color shifts introduced by offaxis viewing for the prototype LCD used in this paper, an additional set of measurements was made for the 64 test 


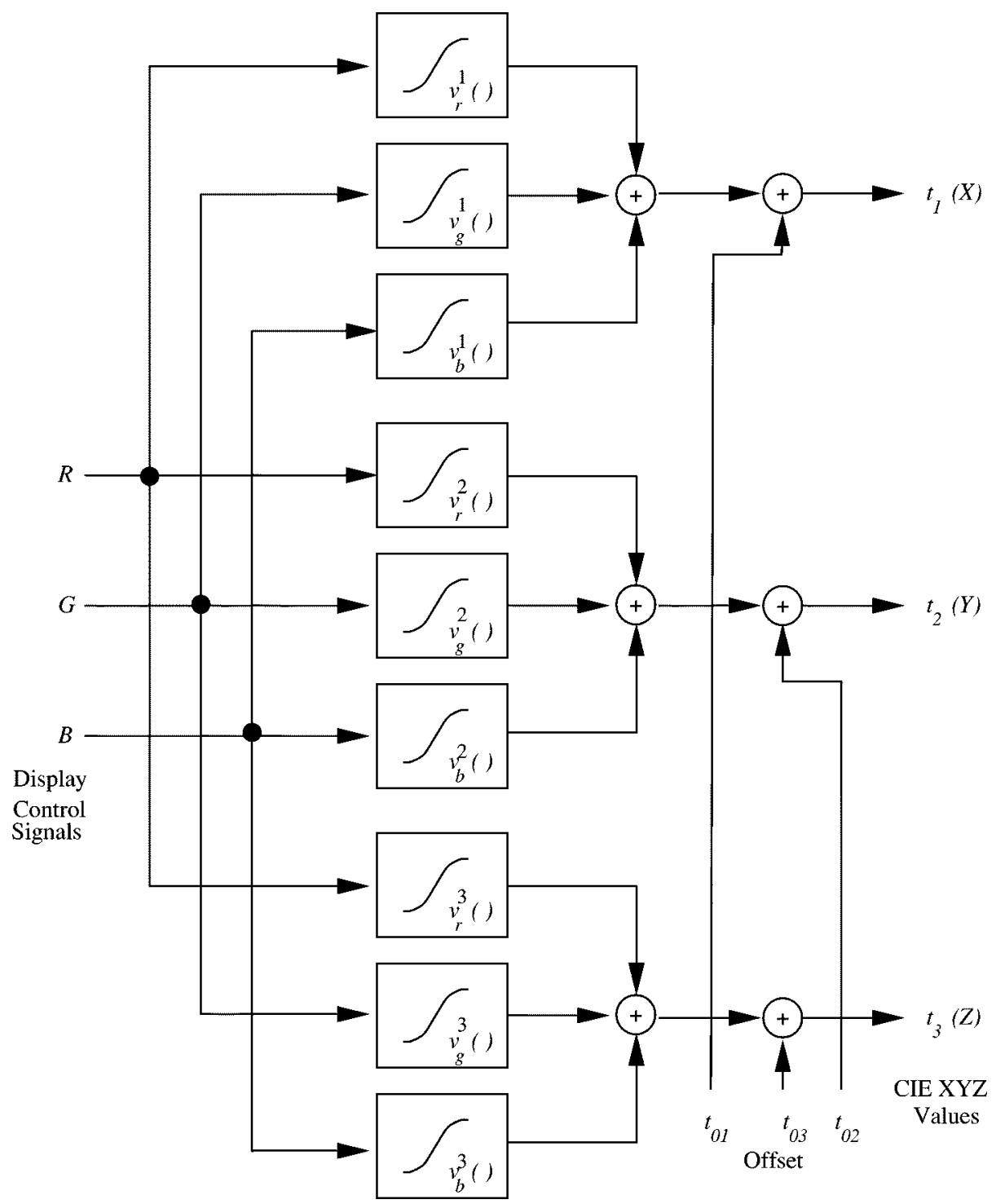

Fig. 12. Graphical representation of the alternate forward model for LCD color calibration.

Table 4

LCD Alternate "Forward Model" Color-Calibration Errors

\begin{tabular}{c|c|c|c|c}
\hline \multirow{2}{*}{ Sample Set } & \multicolumn{3}{|c}{ Color-Calibration Errors } \\
\cline { 2 - 5 } & \multicolumn{2}{|c|}{$\triangle E_{a b}^{*}$ units } & \multicolumn{2}{c}{$\Delta E_{94}^{*}$ units } \\
\cline { 2 - 5 } & Mean & Max. & Mean & Max \\
\hline Characterization RGB ramps & 0.05 & 0.09 & 0.03 & 0.07 \\
\hline Test $4 \times 4 \times 4$ RGB cube & 1.45 & 3.16 & 0.83 & 2.08 \\
\hline
\end{tabular}

patches along a viewing angle of approximately $30^{\circ}$ with respect to the normal to the display. Color differences between the measurements for 0 and $30^{\circ}$ angles were computed in absolute (using the white measurement for $0^{\circ}$ as the white for conversion of both sets of measurements into CIELAB) and in relative (using measured white-point for each case as the white for conversion to CIELAB) CIELAB space. The mean and maximum color differences over the 64 test patches in $\triangle E_{a b}^{*}$ and $\triangle E_{94}^{*}$ units are given in Table 5. For both cases (absolute CIELAB and relative CIELAB), the color differences are quite large and much larger than the color-calibration errors of Section VII. The large magnitude of the dif-
Table 5

Color Shifts Over Test Patches Between 0 and $30^{\circ}$ Viewing for the LCD

\begin{tabular}{|c|c|c|c|c|}
\hline & \multicolumn{4}{|c|}{ Color Shifts } \\
\hline & \multicolumn{2}{|c|}{$\triangle E_{a b}^{*}$ units } & \multicolumn{2}{|c|}{$\triangle E_{94}^{*}$ units } \\
\hline & Mean & Max. & Mean & $\operatorname{Max}$ \\
\hline Absolute CIELAB & 7.94 & 15.03 & 4.53 & 9.24 \\
\hline Relative CIELAB & 5.48 & 11.37 & 2.86 & 8.32 \\
\hline
\end{tabular}

ferences indicates that the LCD should only be used for a limited viewing angle if accurate color is desired.

The color shifts in relative CIELAB space for the 64 test patches are shown in a 3-D plot in Fig. 13. The projections of the color shifts onto the $a^{*} b^{*}$ plane are shown in Fig. 14. The lines in these plots represent the color shift with the dots representing the color seen along a $30^{\circ}$ viewing angle and the other end representing the color that is seen along a $0^{\circ}$ viewing angle. Note that in it is clear that as the viewing angle changes from 0 to $30^{\circ}$, the colors move toward the center of the $a^{*}$ and $b^{*}$ plane. Thus, the color shifts are such that 


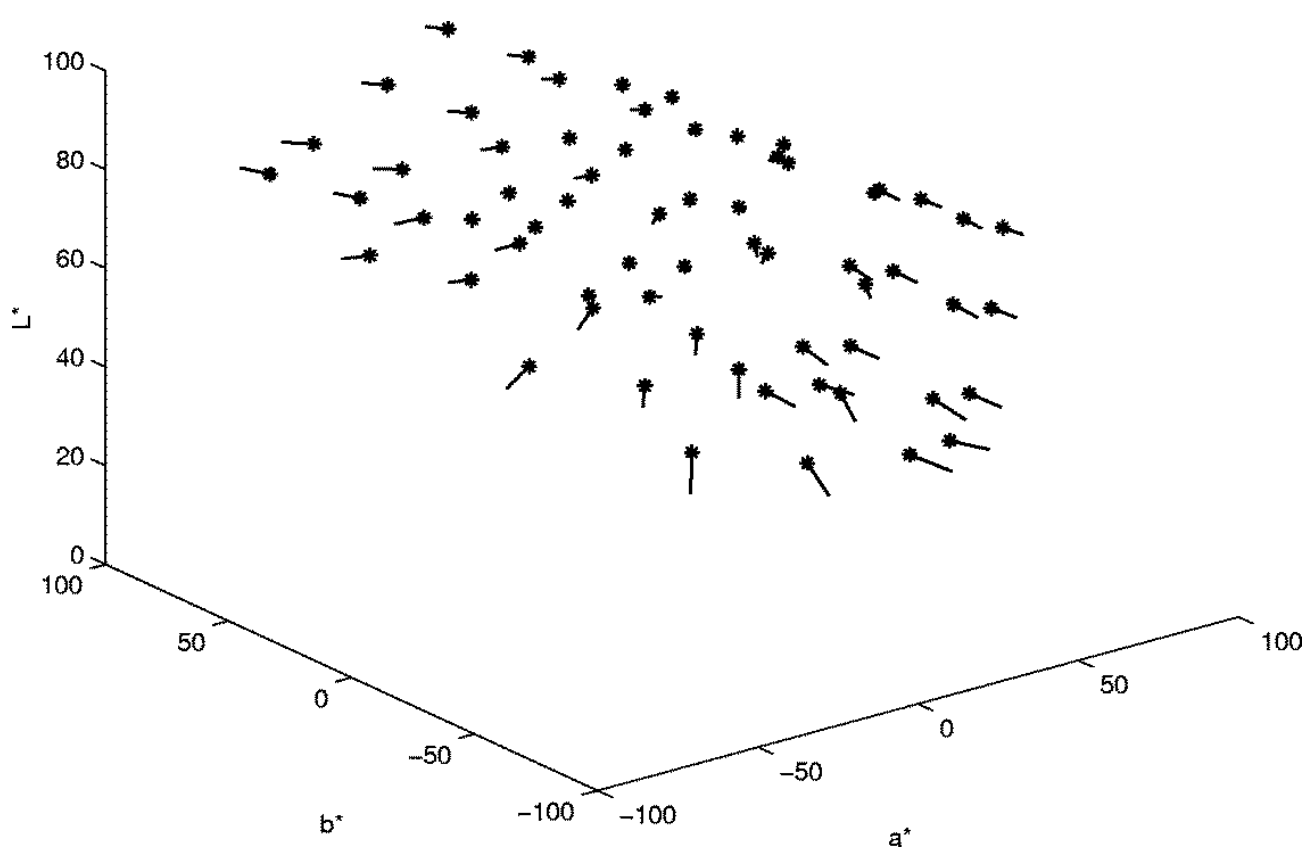

Fig. 13. 3-D plot of color shifts in CIELAB for the LCD test patches for a change in viewing angle from 0 to $30^{\circ}$.

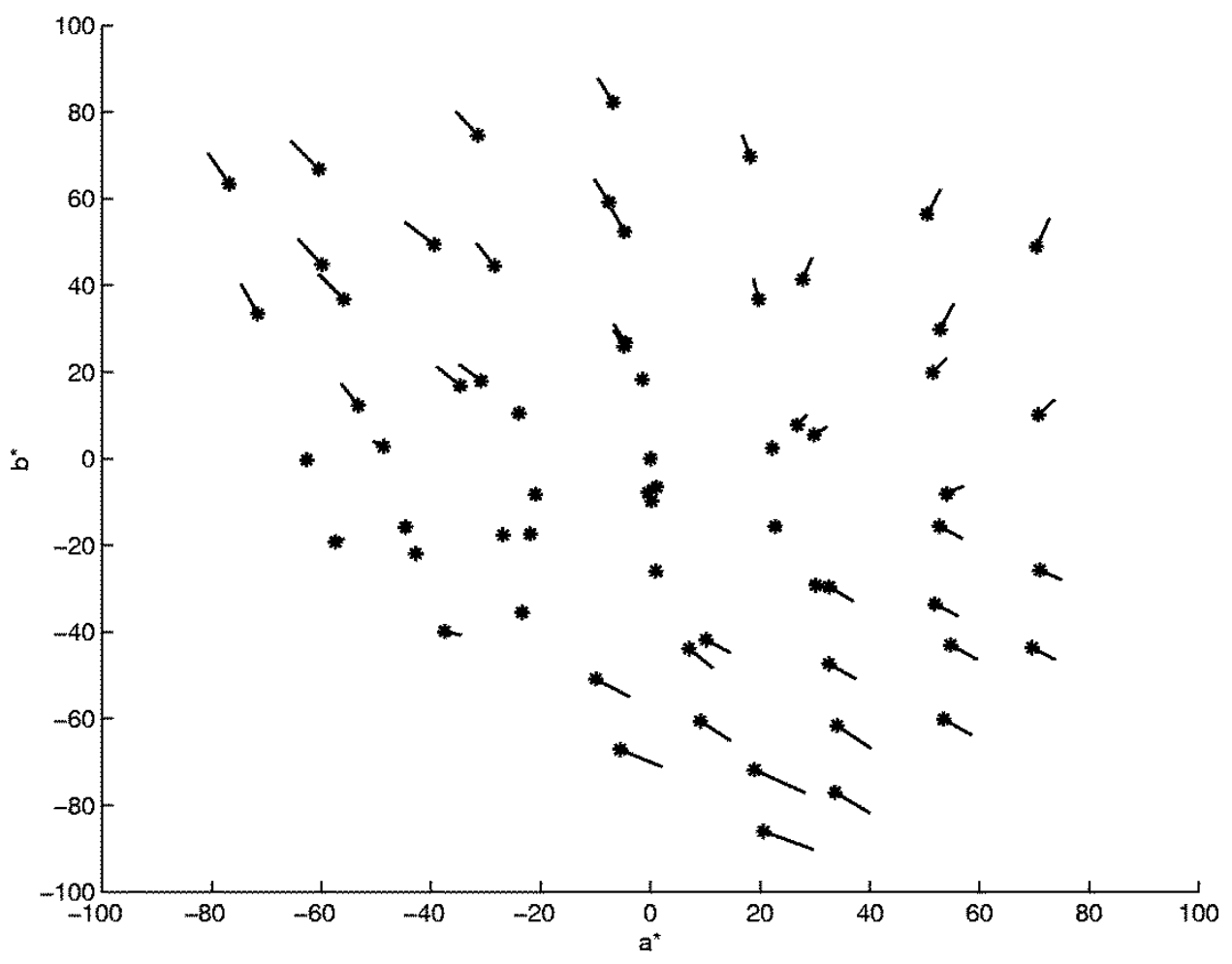

Fig. 14. Color shifts in $a^{*}$ and $b^{*}$ for the LCD test patches for a change in viewing angle from 0 to $30^{\circ}$.

colors tend to desaturate as the viewing angle increases. The predominant effect seen in offaxis viewing is a reduction in contrast and saturation.

\section{COMPARISON OF LCD AND CRT DISPLAYS}

The AMLCD technology has several advantages over the conventional CRT technology. LCDs have smaller size and are less heavy and bulky than the CRTs, which is the driving force for their increasing use in portable and desktop devices. From an image-quality standpoint, the predominant and most clearly visible advantage is the higher spatial resolution of the LCD devices, which translates into sharper images. The color reproduction capabilities and achievable gamut for the LCD and CRT display are critically compared in the remainder of this section. 
1) Color Calibration: From the preceding sections, it is clear that LCDs and CRTs are similar in several respects from the perspective of color calibration. Identical models based on channel independence and channel-chromaticity constancy can be used for the calibration of either type of display and the runtime mapping of images to display color coordinates can also be performed using the inverse model of Fig. 2 in either case. For the CRT, these models provide extremely good accuracy, whereas for LCDs, the accuracy is good enough for most applications. For the CRT, the use of parametric "gamma-offset" models for the individual channel TRCs can further simplify the characterization process and potentially reduce the number of measurements required. The S-shaped TRCs for LCDs are not modeled well by the parametric "gamma-offset" models and, therefore, additional measurements may be required for the characterization of these devices. For the same reason, images that are "gamma-corrected" for display on a CRT will not have the proper tone response if displayed on an LCD, unless appropriate TRCs are used in hardware/software. Scientific applications involving very precise control of the displayed color require more elaborate calibration schemes for the LCD. Note also that due to the significant difference in spectral characteristics of the CRT and LCDs, the impact of chromatic aberration in the eye will be different for the two displays and may need to be compensated when displaying complex images for precise psychophysical experiments [41].

2) Angular Dependence: CRTs are almost Lambertian [18] radiators within typical viewing angles [42], [43] and can, therefore, be viewed over a wide range of viewing angles without loss of contrast or undesirable variations in hue. While many improvements have been made in increasing AMLCD viewing angles, the problem has not been completely eliminated and the useful viewing-angle range of most LCDs is limited in comparison to CRTs. The limited viewing angle of LCDs is often a limitation when precisely color-corrected images are to be displayed before an audience of more than one or two persons.

3) Spatial Homogeneity: LCDs significantly outperform CRTs with regard to spatial homogeneity. While there is negligible variation in the color of a displayed pixel with change in the pixel's position over the screen for an LCD [10], the assumption of spatial homogeneity does not strictly hold for CRTs. In most CRT monitors, for the same driving signals, the light intensity is brightest at the center and falls off toward the edges. The change in luminance over the screen can be as high as $25 \%$ [7, p. 104]. In casual image display applications, this is not as objectionable as measurements would indicate because the eye's sensitivity itself is not uniform over the entire field of view and because the eye adapts well to the smooth variation in intensity across the screen. However, in scientific applications where precise control of the displayed color is required, it is necessary to correct for this spatial inhomogeneity in CRTs [44].

4) Luminance and Dynamic Range: For the displays used in the experiment, the luminance of white on the LCD is about 4.7 times the luminance of white on the CRT monitor. This difference is typical for most LCD and CRT displays [10]. For the measurements made in a completely dark room with almost no additive flare, the luminance of black on the LCD was about 58 times the luminance of black on the CRT and the dynamic range (ratio of white to black luminances) is around $357: 1$ for the LCD and 4351: 1 for the CRT. On the face of it, the CRT appears to have a larger dynamic range. However, in practice, the exact converse is true because a large region of the CRTs dynamic range is lost to additive flare under typical viewing conditions. In the presence of typical viewing flare, the ratio black to white luminance for the CRT falls to $16: 1$, whereas the corresponding ratio for the LCD remains significantly higher at 209:1. This reversal is owing to the fact that the typical viewing flare has a much higher luminance than the CRT black in a dark room but is quite negligible as compared to the light leakage through the LCD cells already present in the LCD black. The higher white luminance for the LCDs gives them a higher effective dynamic range than typical CRTs, which is clearly apparent in practice.

5) Intrinsic Gray Balance: It was observed in Section VI that individual red-, green-, and blue-channel TRCs of the CRT display were fairly close while those for the LCD were not. The difference between the LCD RGB TRCs would imply that a "device gray wedge" along $R=G=B$ would not appear visually neutral (gray balanced) when displayed on the LCD, but would appear almost neutral when displayed on the CRT. This is actually observed in practice. Since graphics programs often create images or sweeps directly in display device color space, it is desirable to have the display gray-balanced and the CRTs characteristics are, therefore, preferable. Note, however, that this limitation of the LCD is easily overcome once the display is calibrated and the inverse TRCs are incorporated into the video path.

6) Channel Chromaticities: Fig. 15 shows the location of the channel chromaticities (the end points of the respective triangles) for the CRT and the LCD in relation to the spectrum locus on the CIE xy chromaticity diagram [16]-[18]. Note that the red channel chromaticity for the CRT and the LCD are fairly close to each other on the chromaticity diagram, but the blue and green channel chromaticities are different from each other. Also plotted on the same diagram are the chromaticities for the white point for the CRT (labeled as letter C on the plot), the LCD (L), and the CIE D50 and D65 daylight illuminants. Note that the LCD white point is somewhere between the D65 and D50 white points, while the CRT white point is close to D65 in chromaticity, which agrees with the selected $6500-\mathrm{K}$ color temperature for the CRT. The differences in white point and in the channel chromaticities imply that the 3 $\times 3$ color calibration matrices for the LCD and the CRT display in the model of Fig. 2 are different. This implies that transformation of an image in CRT RGB coordinates to LCD RGB coordinates requires full color correction and cannot be achieved by using 1-D corrections for each of the channels. While only one LCD was considered in the experiment of this paper, this implication is probably true 


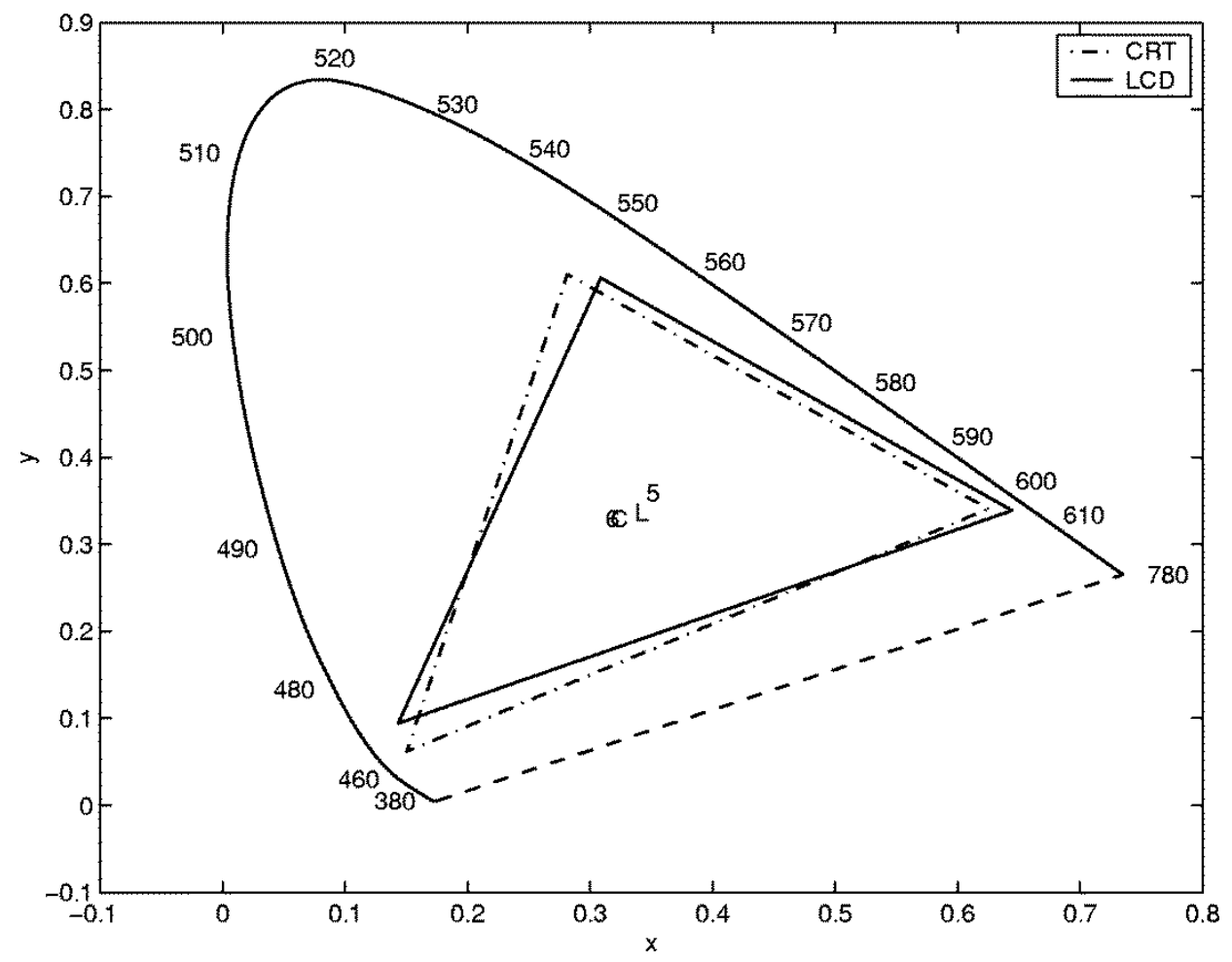

Fig. 15. Channel chromaticities for the LCD and CRT displays in relation to the spectrum locus. Also shown are the white-points C (CRT), L (LCD), 6 (CIE D65), and 5 (CIE D50).

in general because there are bound to be variations in LCD channel chromaticities due to manufacturing tolerances in the fabrication of the LCD filters and backlights.

7) Achievable Color Gamut: The triangle formed by the three-channel chromaticity-coordinates for each display in Fig. 15 represents the achievable gamut for the display in chromaticity space. The 2-D representation is however rather limited and comparisons of the 3 -D gamut in CIELAB coordinates using visualization tools [45] provide a much more complete and useful picture. Since the perception of color is significantly influenced by the viewing conditions [46], three different gamut comparisons were performed, corresponding to different assumptions for the viewing conditions: 1) comparisons of ideal flareless "relative" gamuts, where each monitors own white point was used as the nominal white-point [18, p. 167] in the conversion from CIEXYZ to CIELAB; 2) comparison of "relative" gamuts with typical flare; and 3) comparison of "absolute" gamuts, where flare is included and the CIEXYZ values for the LCD white (having the higher luminance) were used in the CIEXYZ to CIELAB conversion for both the measurements from the LCD and the CRT. The first case represents the ideal achievable gamuts, assuming that the displays are viewed individually in a dark room and that the photopic response of the eye extends until the black point of the CRT (which does not really hold). The second case corresponds to the more typical situation when the two displays are viewed individually at different times in typical viewing environments. The third case is representative of the scenario when the two displays are viewed side by side at the same time (the assumption here is that when the viewer sees the two displays side by side, he/she adapts to the brighter white). For each of the three cases, the 3-D gamuts were computed in CIELAB space and visualized simultaneously with the larger gamut shown as a wire mesh and the smaller gamut as a solid. Figs. 16-18 show perspective views of the gamuts for these different cases, where each figure includes a top view looking down on the gamuts from the $L^{*}$ axis and a side view, whose perspective position was chosen to highlight the gamut differences.

For the flareless idealized gamuts of Fig. 16, the CRT gamut (wire frame) and the LCD gamut (solid) are fairly close in most color regions, except in the blue and magenta regions of color space, where the CRT gamut extends further outwards covering a larger volume. The CRT gamut also extends further outward than the LCD gamut in the dark regions close to black. In the presence of typical viewing flare, however, the CRT not only loses its advantage over the LCD, but also ends up with significantly smaller gamut in the dark regions. This can be seen from the gamut comparisons of Fig. 17, where the LCD gamut (wire frame) is significantly larger than the CRT gamut (solid), particularly in the dark color regions around black. The dramatic increase in lightness $\left(L^{*}\right)$ value for the CRT black between Figs. 16 and 17 is indicative of the significant reduction in dynamic range caused by flare, which was mentioned earlier. The significantly larger gamut in the dark color regions provides the LCDs a significant advantage over CRTs when displaying images with large dynamic range and shadow detail. Fig. 18 presents a comparison of the LCD (wire frame) and CRT (solid) gamuts in "absolute" CIELAB (Case 3, above). The "absolute" CRT gamut is almost entirely contained 


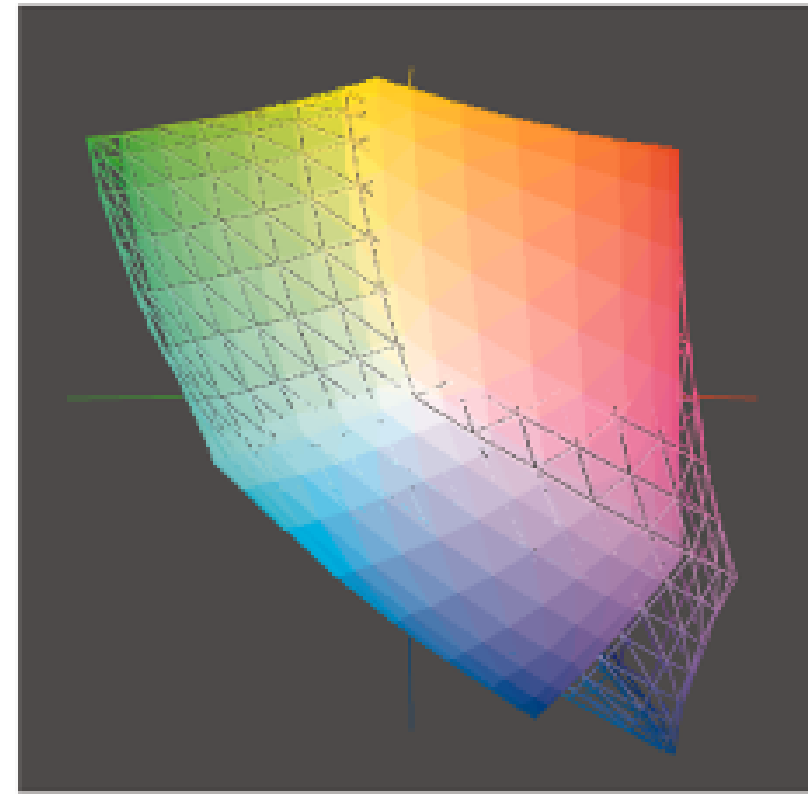

(a)

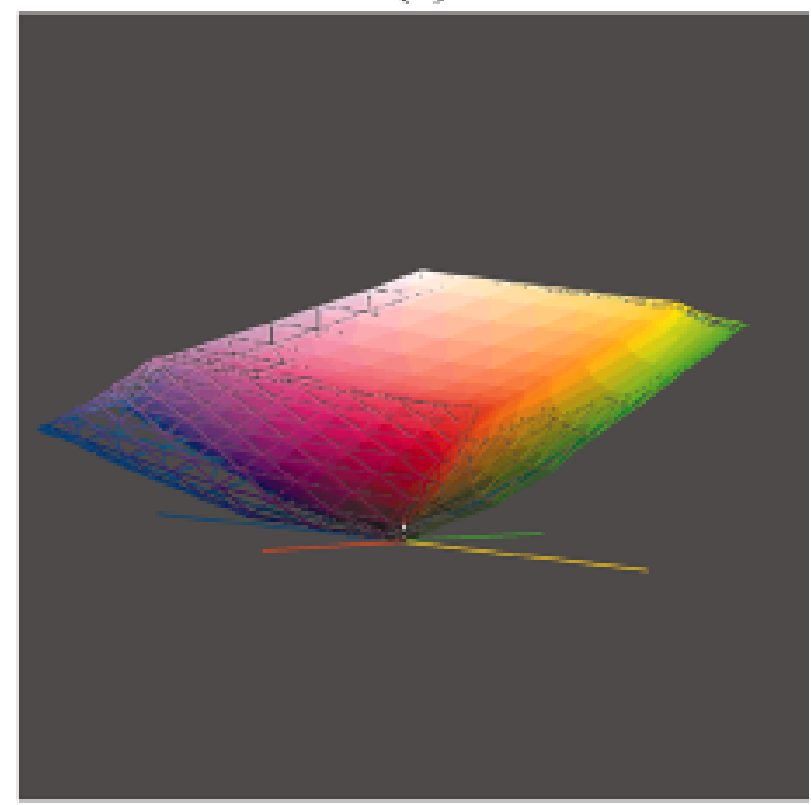

(b)

Fig. 16. Comparison of flareless relative CIELAB gamuts of an LCD (solid) and a CRT (wire frame). (a) Top view. (b) Side view.

inside the LCD gamut and quite small in comparison. This is primarily due to the much higher luminances of the LCD. These gamut comparisons also illustrate why the CRT appears quite satisfactory when viewed by itself independently of the LCD (gamut comparisons of Fig. 17), but seems to be quite "washed out" when viewed side by side with the LCD (gamut comparisons of Fig. 18).

\section{CONCLUSION}

AMLCD color displays can be color-calibrated with good accuracy (average color error of approx. $1 \triangle E_{94}^{*}$ ) by using a

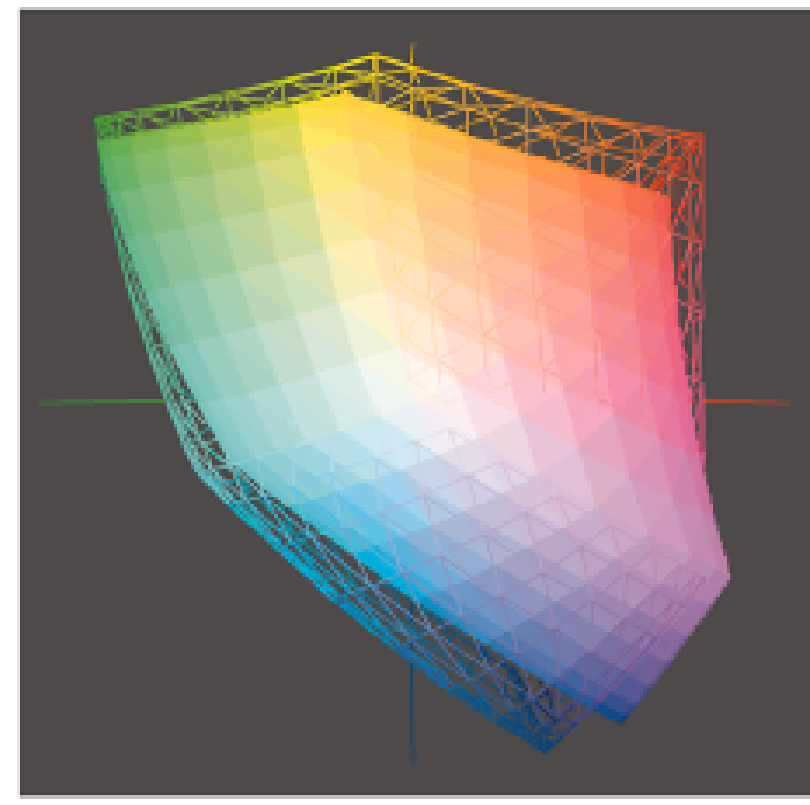

(a)

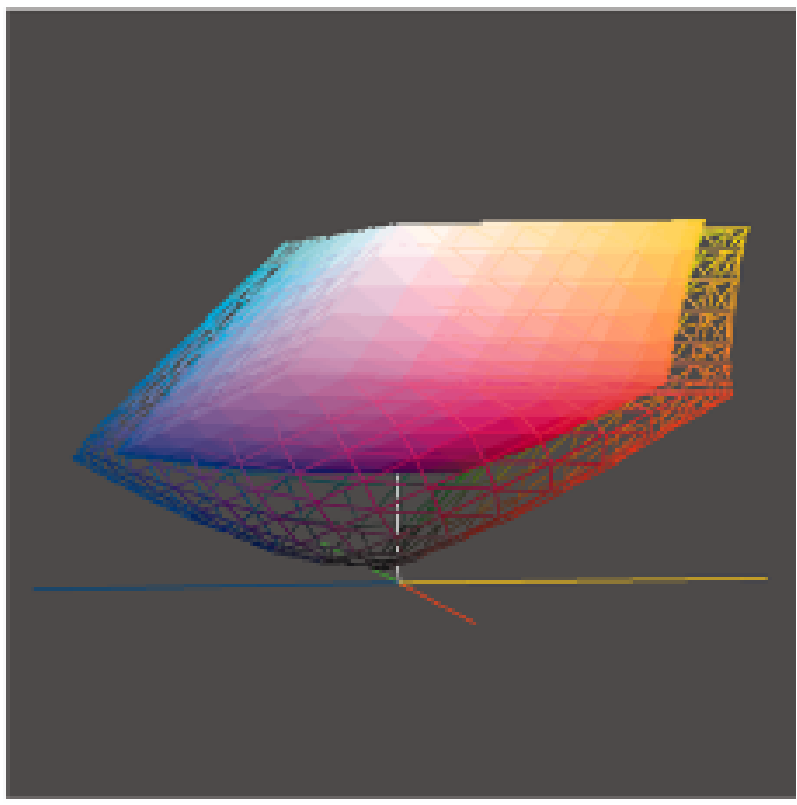

(b)

Fig. 17. Comparison of relative CIELAB gamuts under normal viewing flare conditions for an $\mathrm{LCD}$ (wire frame) and a CRT (solid). (a) Top view. (b) Side view.

simple offset, matrix, and tone-response correction for the conversion of tristimulus values into display RGB. If a LUT is to be used for color correction, the accuracy of the characterization can be increased without requiring additional measurements by using an alternate model that does not assume constancy of chromaticity for each channel. The calibration accuracy is lower than what is achieved by CRTs, but is acceptable for most applications. The raw TRCs for LCDs are S-shaped and are not accurately represented by the "gamma+offset" parametric model commonly used for CRTs. Display calibration techniques that are based on these 


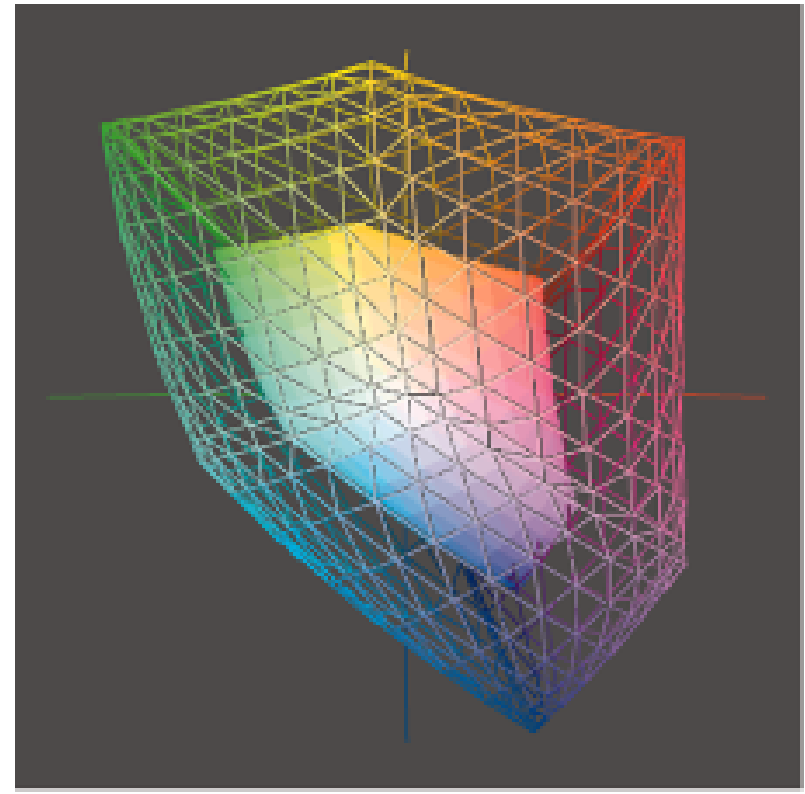

(a)

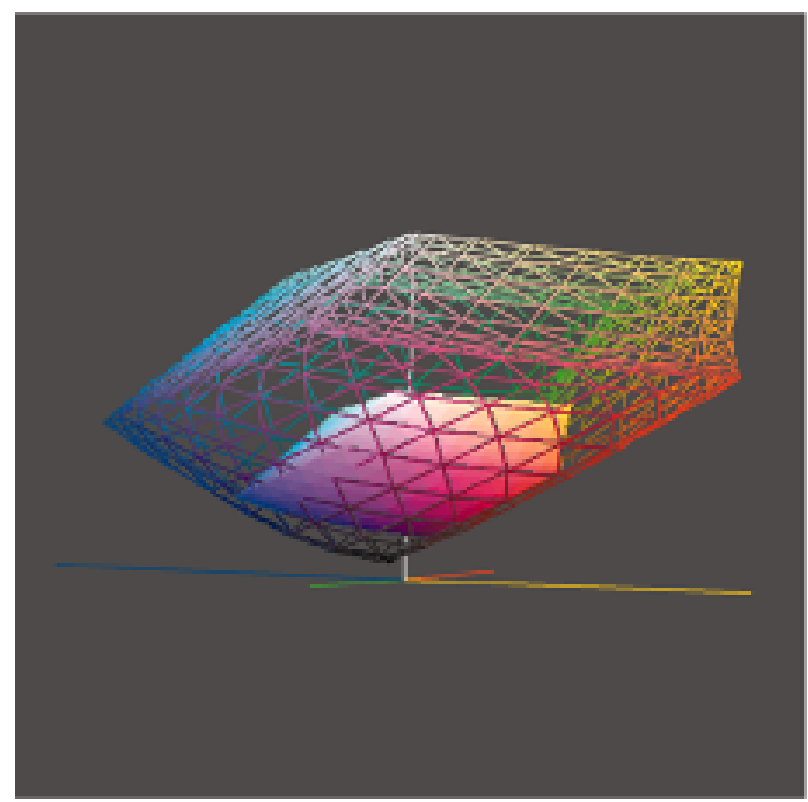

(b)

Fig. 18. Comparison of "absolute" CIELAB gamuts of an LCD (wire frame) and a CRT (solid). (a) Top view. (b) Side view.

parametric models should not be directly applied to LCD color calibration.

The difference between the uncorrected TRCs for AMLCDs and CRTs implies that images designed for CRTs will not have the proper tone response on these displays, unless appropriate tone-response corrections are used in hardware/software. The LCD channel-chromaticity coordinates are determined by the backlight and color filter spectral characteristics, and may not correspond to the chromaticity coordinates for commonly employed CRT phosphors. Therefore, a simple per-channel 1-D correction cannot be used to universally map CRT RGB to LCD RGB.
The prototype display studied in this paper showed a strong variation in color with change in viewing angle. This viewing-angle dependence limits the utility of the display in accurate color demonstrations where the display is to be simultaneously viewed by multiple observers. While significant viewing-angle improvements have been made in commercial displays [10], further improvements are still needed to match CRT viewing angles.

Typical AMLCDs possess a significantly larger gamut than typical CRT displays, with the AMLCD gamut extending significantly beyond CRT gamut in the dark color regions. The differences in gamut arise primarily due to the higher luminance of LCDs and provide AMLCDs a significant advantage over CRTs in the reproduction of images with high dynamic range and shadow detail.

\section{REFERENCES}

[1] S. L. Wright, R. W. Nywening, S. E. Millman, J. Larimer, J. Gille, and J. Luszcz, "Image quality issues for height resolutions TFTLCDs," in Proc. IS\&T/SID 7th Color Imaging Conf., Scottsdale, AZ, Nov. 1999, pp. 100-105.

[2] J. A. Castellano, "Trends in the global CRT market," in SID Int. Symp. Dig. , May 1999, pp. 356-359.

[3] K. I. Werner, "The flat panel's future," IEEE Spectrum, vol. 30, pp. 18-26, Nov. 1993.

[4] W. B. Cowan, "An inexpensive scheme for calibration of a color monitor in terms of standard CIE coordinates," Comput. Graphics, vol. 17, pp. 315-321, July 1983 .

[5] W. B. Cowan and N. Rowell, "On the gun independency and phosphor constancy of color video monitors," Color Res. Appl., vol. 11, pp. S34-S38, 1986.

[6] D. H. Brainard, "Calibration of a computer controlled color monitor," Color Res. Appl., vol. 14, no. 1, pp. 23-34, Feb. 1989.

[7] V. Mani, "Calibration of color monitors," M. S. thesis, North Carolina State University, Raleigh, NC, 1991.

[8] R. S. Berns, R. J. Motta, and M. E. Gorzynski, "CRT colorimetry_Part I: Theory and practice," Color Res. Appl., vol. 18, no. 5, pp. 299-314, Oct. 1993.

[9] R. S. Berns, M. E. Gorzynski, and R. J. Motta, "CRT colorimetry_Part II: Metrology," Color Res. Appl., vol. 18, no. 5, pp. 315-325, Oct. 1993.

[10] M. D. Fairchild and D. Wyble, "Colorimetric characterization of the apple studio display (flat panel LCD)," Munsell Color Science Lab., Rochester Institute of Technology, Rochester, NY, Tech. Rep., July 1998.

[11] C. Y. Tsai, M. J. Shaw, and H. P. D. Shieh, "Color characterization and reproduction of TN-LCDs," in SID Int. Symp. Dig., May 1999, pp. 790-797.

[12] G. Sharma, "Color calibration of DPiX AMLCD display," Xerox Corp., Webster, NY, Internal Memo, May 1999.

[13] R. Martin, J. Batey, T. Fiske, M. Nguyen, E. Rabner, D. Siemens, H. Steemers, S. Stuber, M. Thompson, W. Turner, M. Tilton, L. D. Silverstein, and M. Potts, "Design of high-resolution AMLCDs with greater than 2000 gate lines," in SID Int. Symp. Dig., May 1997, pp. $7-10$.

[14] M. J. Vrhel and H. J. Trussell, "Color device calibration: A mathematical formulation," IEEE Trans. Image Processing, vol. 8, pp. 1796-1806, Dec. 1999.

[15] "Colorimetry," Central Bureau of the CIE, Vienna, Austria, CIE Publication No. 15.2, 1986.

[16] G. Sharma and H. J. Trussell, "Digital color imaging," IEEE Trans. Image Processing, vol. 6, pp. 901-932, July 1997.

[17] G. Sharma, M. J. Vrhel, and H. J. Trussell, "Color imaging for multimedia," Proc. IEEE, vol. 86, pp. 1088-1108, June 1998.

[18] G. Wyszecki and W. S. Stiles, Color Science: Concepts and Methods, Quantitative Data and Formulae, 2nd ed. New York: Wiley, 1982.

[19] C. D. Child, "Discharge from hot CaO," Phys. Rev., vol. 32, no. 5, pp. 492-511, May 1911

[20] I. Langmuir, "The effect of space charge and residual gases on thermionic current in high vacuum," Phys. Rev., Second Series, vol. 2, no. 6, pp. 450-486, Dec. 1913. 
[21] B. M. Oliver, "Tone rendition in television," Proc. IRE, vol. 38, pp. 1288-1300, 1950.

[22] W. N. Sproson, Color Science in Television and Display Systems. Bristol, U.K.: Adam Hilger, 1983.

[23] C. A. Poynton, "Gamma correction and tone reproduction in scanned photographic images," SMPTE J., vol. 103, pp. 377-385, June 1994.

[24] F. Kretz, "Subjectively optimal quantization of pictures," IEEE Trans. Commun., vol. COM-23, no. 11, pp. 1288-1292, Nov. 1975.

[25] J. M. Kasson and W. Plouffe, "An analysis of selected computer interchange color spaces," ACM Trans. Graphics, vol. 11, no. 4, pp. 373-405, Oct. 1992.

[26] C. A. Poynton, "'Gamma' and its disguises: The nonlinear mappings of intensity in perception, CRTs, film, and video," SMPTE J., vol. 102, pp. 1099-1108, Dec. 1993.

[27] R. Patterson, "Gamma correction and tone reproduction in scanned photographic images," SMPTE J., vol. 103, pp. 377-385, June 1994.

[28] R. W. G. Hunt, The Reproduction of Color, 3rd ed. New York: Wiley, 1975.

[29] M. Anderson, R. Motta, S. Chandrasekar, and M. Stokes, "Proposal for a standard default color space for the internet—sRGB," in Proc. IS\&T/SID 4th Color Imaging Conf., Scottsdale, AZ, Nov. 1996, pp. 238-246.

[30] M. A. Karim, Ed., Electro-Optical Displays. New York: Marcel Dekker, 1992.

[31] L. E. Tannas, Ed., Flat Panel Displays and CRTs. New York: Van Nostrand Reinhold, 1985.

[32] , "Industrial color difference evaluation," Central Bureau of the CIE, Vienna, Austria, CIE Publication No. 116-1995, 1995.

[33] R. J. Motta, "Visual characterization of color CRTs," Proc. SPIE, vol. 1909, pp. 212-221, Feb. 1993.

[34] N. Katoh and T. Deguchi, "Reconsideration of the CRT monitor characteristics," in Proc. IS\&T/SID 5th Color Imaging Conf., Scottsdale, AZ, Nov. 1997, pp. 33-40.

[35] T. Deguchi, N. Katoh, and R. S. Berns, "Colorimetric characterization of CRT monitors," in SID Int. Symp. Dig., May 1999, pp. 786-789.

[36] T. G. Fiske and L. D. Silverstein, "Characterizations of viewingangle-dependent colorimetric and photometric performance of color LCDs," in SID Int. Symp. Dig., May 1999, pp. 565-568.

[37] S. Kaneko, Y. Hirai, and K. Sumiyoshi, "Wide-viewing angle improvements for AMLCDs," in SID Int. Symp. Dig., May 1999, pp. $265-268$.

[38] J. Pollack, "Sharp Microelectronics' approach to new-generation AMLCDs," Inform. Display, vol. 15, no. 2, pp. 16-20, Feb. 1999.

[39] C. Heinmüller, G. Haas, and P. M. Knoll, "Design of in-plane-compensation foils for viewing-angle enhancement," in SID Int. Symp. Dig., May 1999, pp. 90-93.
[40] J. Chenand, K. C. Kang, J. DelPico, H. Seiberle, and M. Schadt, "Wide-viewing-angle photoaligned plastic films for TN-LCDs,", in SID Int. Symp. Dig., May 1999, pp. 98-101.

[41] D. H. Marimont and B. A. Wandell, "Matching color images: The impact of axial chromatic aberration," J. Opt. Soc. Amer. A, vol. 11, no. 12, pp. 3113-3122, Dec. 1994.

[42] J. Kalmer, Luminescent Screens: Photometry and Colorimetry. London, U.K.: Iliffe, 1969.

[43] K. Kinameri, "Photometric error due to light emitting properties of color TV picture tubes," J. Illum. Eng. Inst. Japan, vol. 67, pp. 74-79, 1983.

[44] J. N. Cook, P. A. Sample, and R. N. Weinreb, "Solution to spatial inhomogenity on video monitors," Color Res. Appl., vol. 18, no. 5, pp. 334-340, Oct. 1993.

[45] R. Rolleston, "Visualization of colorimetric calibration," Proc. SPIE, vol. 1912, pp. 299-309, Feb. 1993.

[46] M. D. Fairchild, Color Appearance Models. Reading, MA: Addison-Wesley, 1998.

[47] SID Int. Symp. Dig., May 1999.

[48] SID Int. Symp. Dig., May 1993.

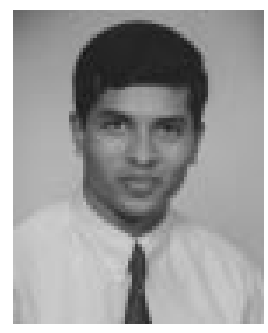

Gaurav Sharma (Senior Member, IEEE) received the B.E. degree in electronics and communication engineering from University of Roorkee, India, in 1990, the M.E. degree in electrical communication engineering from the Indian Institute of Science, Bangalore, India, in 1992, and the M.S. degree in applied mathematics and the Ph.D. degree in electrical and computer engineering from North Carolina State University (NCSU), Raleigh, in 1995 and 1996, respectively.

From August 1992 through August 1996, he was a Research Assistant with the Center for Advanced Computing and Communications in the Electrical and Computer Engineering Department, NCSU. Since August 1996, he has been a Member of the Research and Technical Staff with the Xerox Corporation's Digital Imaging Technology Center, Webster, NY. He is also an Adjunct with the Electrical and Computer Engineering Departments at the Rochester Institute of Technology, Rochester, NY. His current research interests include color science and imaging, signal restoration, image security and halftoning, and error correction coding.

Dr. Sharma is a Member of Sigma Xi, Phi Kappa Phi, Pi Mu Epsilon. He is the Vice-President of the Rochester Chapter of the IEEE Signal Processing Society. 